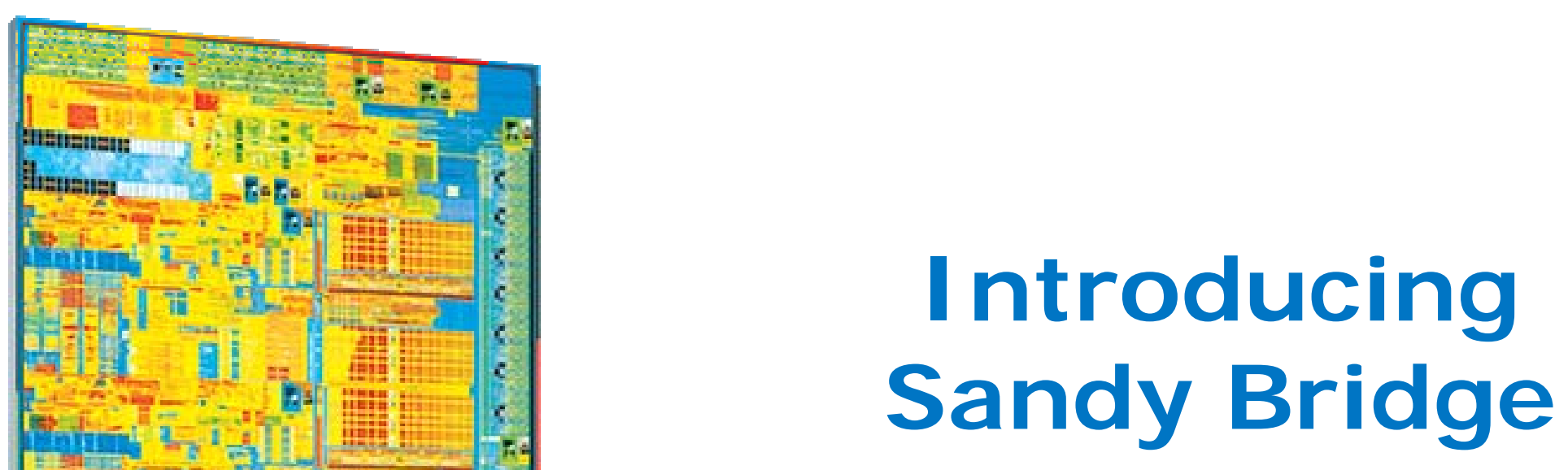

Bob Valentine

Senior Principal Engineer

\title{
Sandy Bridge
}




\section{Sandy Bridge: Overview}

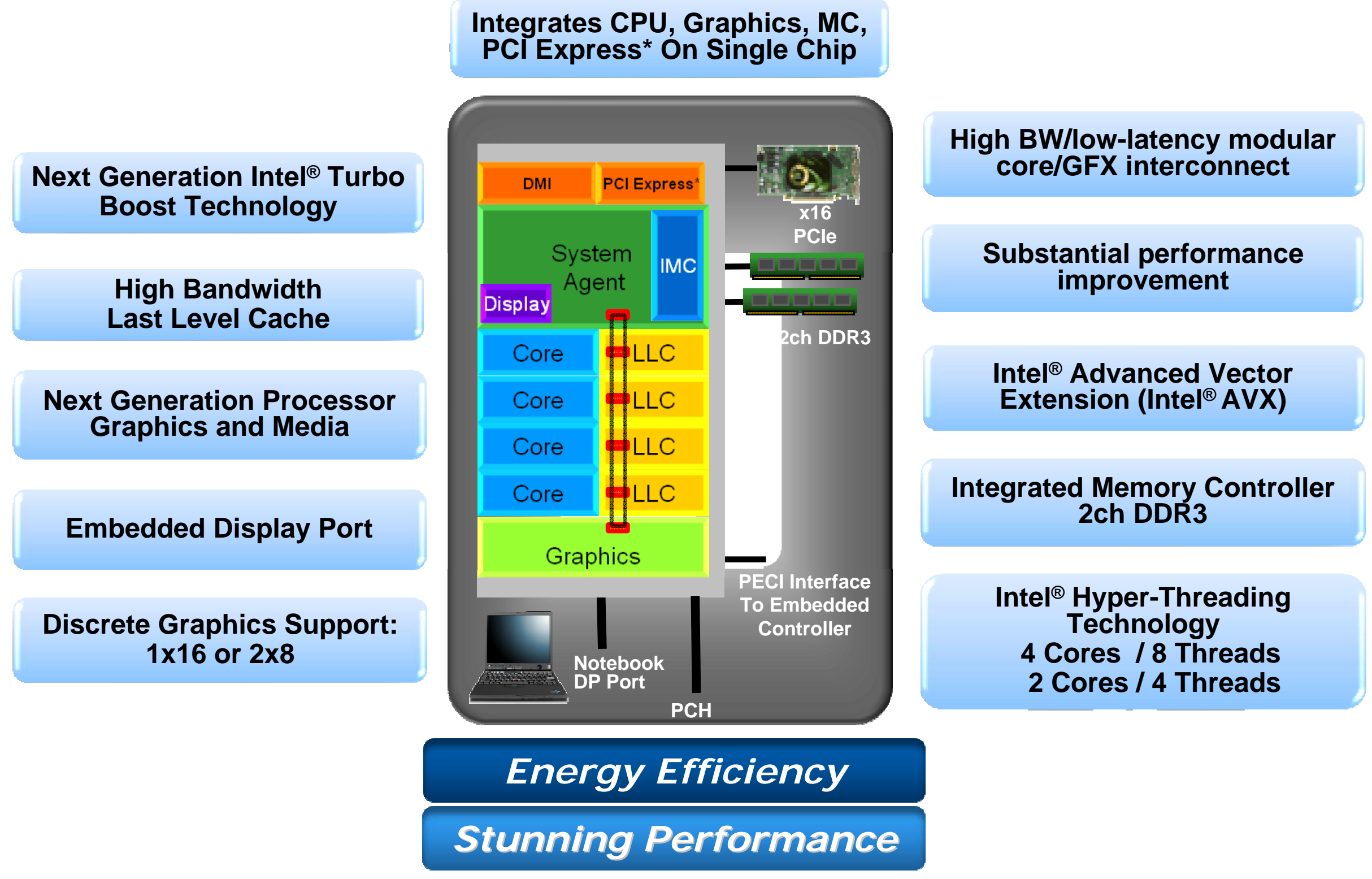




\section{Agenda}

- Innovation in the Processor core

- System Agent, Ring Architecture and Other Innovations

- Innovation in Power Management 
Sandy Bridge

Microarchitecture

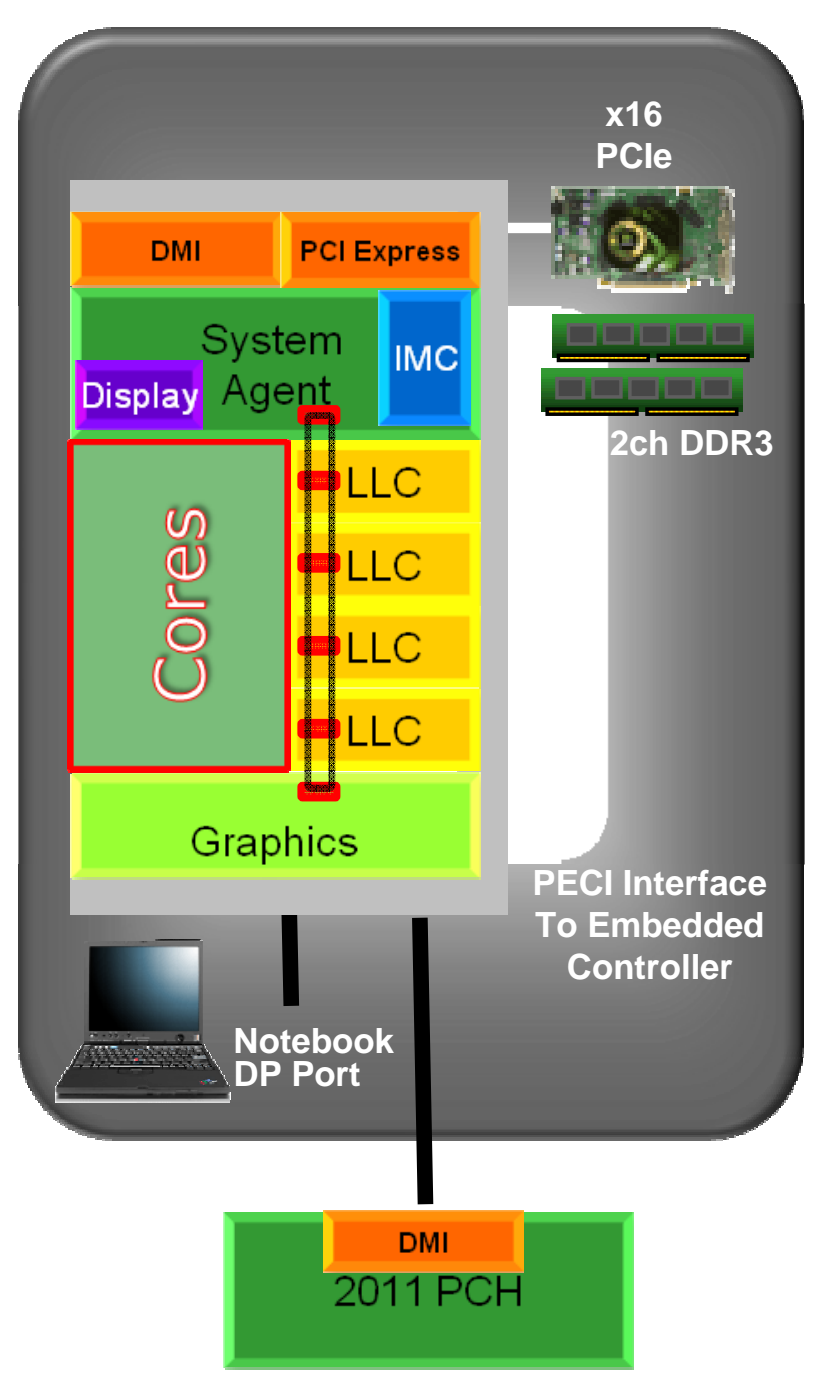

\section{I ntroduction to Sandy Bridge Processor Core Microarchitecture}




\section{Outline}

- Sandy Bridge Processor Core Summary

- Core Major Microarchitecture Enhancements

- Core Architectural Enhancements

- Processor Core Summary 


\section{Sandy Bridge Processor Core Summary}

- Build upon the successful Nehalem microarchitecture processor core

- Converged building block for mobile, desktop, and server

- Add "Cool" microarchitecture enhancements

- Features that are better than linear performance/power

- Add "Really Cool" microarchitecture enhancements

- Features which gain performance while saving power

- Extend the architecture for important new applications

- Floating Point and Throughput

- Intel ${ }^{\circledR}$ Advanced Vector Extensions (Intel ${ }^{\circledR}$ AVX) - Significant boost for selected compute intensive applications

- Security

- AES (Advanced Encryption Standard) throughput enhancements

- Large Integer RSA speedups

- OS/VMM and server related features

- State save/restore optimizations 


\title{
Processor Core Tutorial Microarchitecture Block Diagram
}

\author{
Front End \\ ( I A instructions $\rightarrow$ Uops)
}

In Order Allocation, Rename, Retirement

Out of Order "Uop" Scheduling

Six Execution Ports

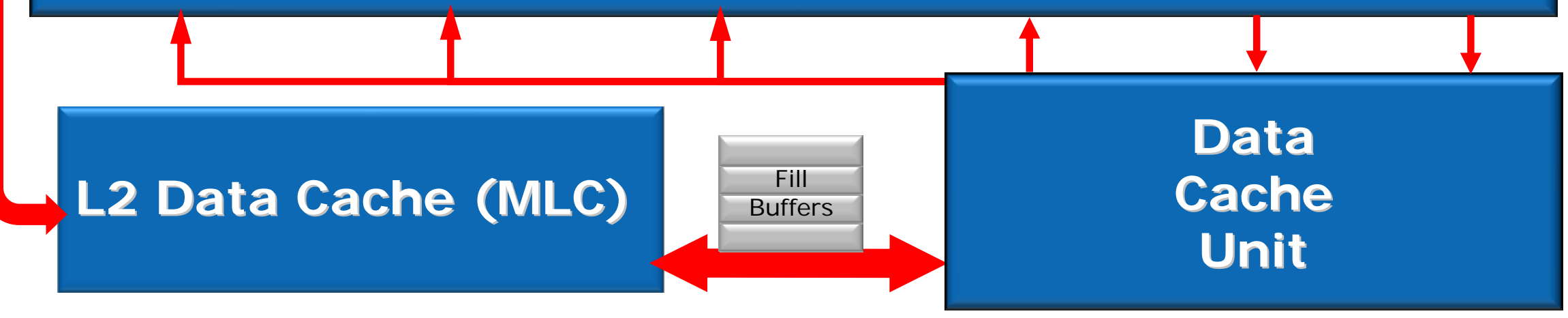




\section{Front End Microarchitecture}

Instruction Decode in Processor Core

- 32 Kilo-byte 8-way Associative ICache

- 4 Decoders, up to 4 instructions / cycle

- Micro-Fusion

- Bundle multiple instruction events into a single "Uops"

- Macro-Fusion

- Fuse instruction pairs into a complex "Uop"

- Decode Pipeline supports 16 bytes per cycle 


\section{New: Decoded Uop Cache}
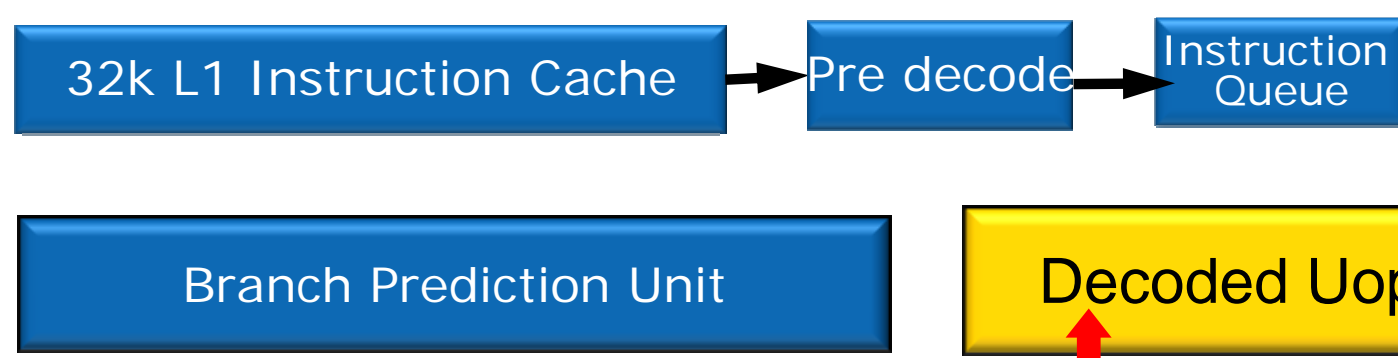

\section{Decoders}

Branch Prediction Unit

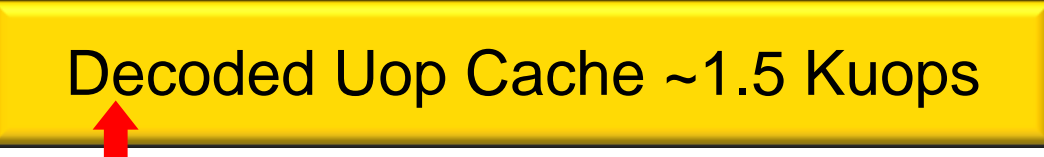

\section{Add a Decoded Uop Cache}

- An LO Instruction Cache for Uops instead of Instruction Bytes

- $\sim 80 \%$ hit rate for most applications

- Higher Instruction Bandwidth and Lower Latency

- Decoded Uop Cache can represent 32-byte / cycle

- More Cycles sustaining 4 instruction/cycle

- Able to 'stitch' across taken branches in the control flow 


\section{New Branch Prediction Unit}

32k L1 Instruction Cache

Branch Prediction Unit

Do a 'Ground Up' Rebuild of Branch Predictor

- Twice as many targets

- Much more effective storage for history

- Much longer history for data dependent behaviors 


\section{Sandy Bridge Front End Microarchitecture}

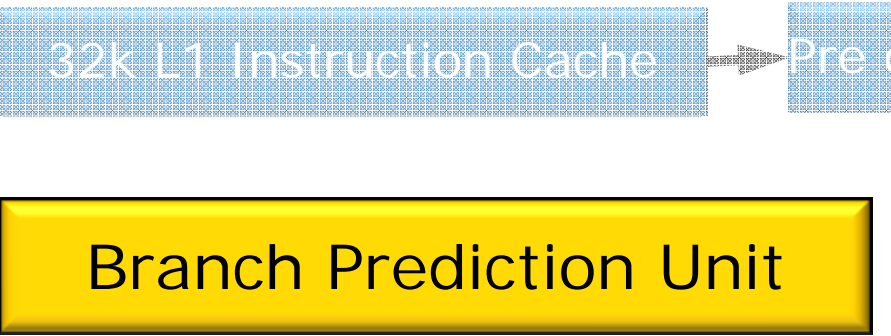

Decoded Uop Cache $~ 1.5$ Kuops

"Really Cool" features in the front end

- Decoded Uop Cache lets the normal front end sleep

- Decode one time instead of many times

- Branch-Mispredictions reduced substantially

\section{"Really Cool" Features}

Save Power while Increasing Performance

Power is fungible...

.give it to other units in this core, or other units on die 


\section{"Out of Order" Cluster}

In Order Allocation, Rename, Retirement

\section{Out of Order "Uop" Scheduling}

- Receives Uops from the Front End

- Sends them to Execution Units when they are ready

- Retires them in Program Order

- Goal: Increase Performance by finding more Instruction Level Parallelism

- Increasing Depth and Width of machine implies larger buffers

- More Data Storage, More Data Movement, More Power

Challenge to the $\mathrm{OoO}$ Architects :

Increase the ILP while keeping the power available for Execution 


\section{Sandy Bridge Out-of-Order (000) Cluster}

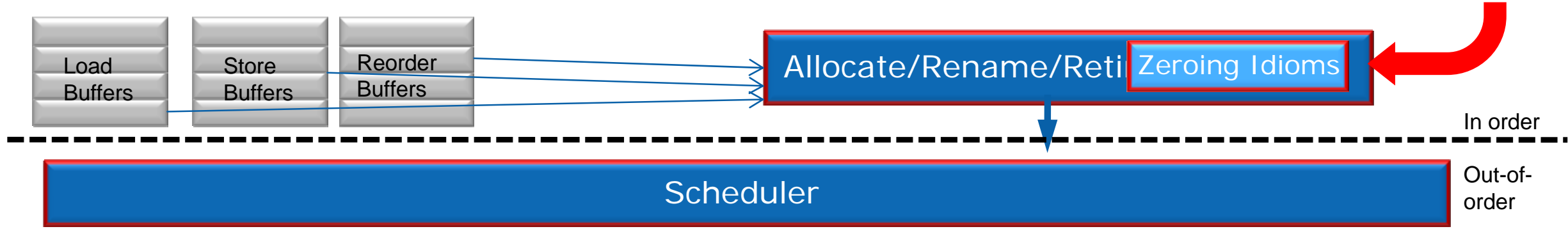

FP/ I NT Vector PRF

\section{Int PRF}

- Method: Physical Reg File (PRF) instead of centralized Retirement Register File

- Single copy of every data

- No movement after calculation

- Allow significant increase in buffer sizes

- Dataflow window $\sim 33 \%$ larger

PRF is a "Cool" feature better than linear performance/ power

Key enabler for Intel ${ }^{\circledR}$ Advanced Vector Extensions (Intel ${ }^{\circledR}$ AVX)

\begin{tabular}{|l|c|c|}
\hline & Nehalem & $\begin{array}{c}\text { Sandy } \\
\text { Bridge }\end{array}$ \\
\hline Load Buffers & 48 & 64 \\
\hline Store Buffers & 32 & 36 \\
\hline $\begin{array}{l}\text { RS - Scheduler } \\
\text { Entries }\end{array}$ & 36 & 54 \\
\hline PRF integer & N/A & 160 \\
\hline PRF float-point & N/A & 144 \\
\hline ROB Entries & 128 & 168 \\
\hline
\end{tabular}




\section{Execution Cluster}

- 3 Execution Ports

- Maximum throughput of 8 floating point operations* per cycle

- Port 0 : packed SP multiply

- Port 1 : packed SP add

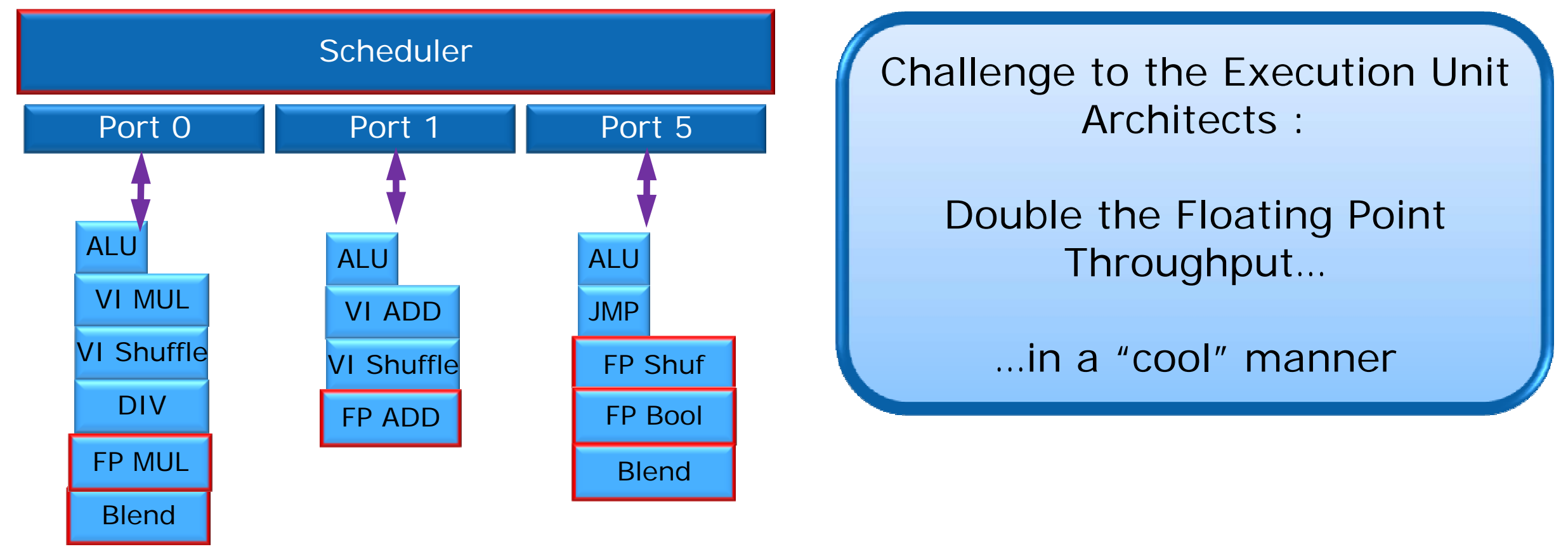

${ }^{*}$ FLOPS $=$ Floating Point Operations $/$ Second 


\section{Doubling the FLOPs in a "Cool" Manner}

- Intel ${ }^{\circledR}$ Advanced Vector Extensions (Intel ${ }^{\circledR}$ AVX)

- Extend SSE FP instruction set to 256 bits operand size

- Intel AVX extends all 16 XMM registers to 256bits

- New, non-destructive source syntax

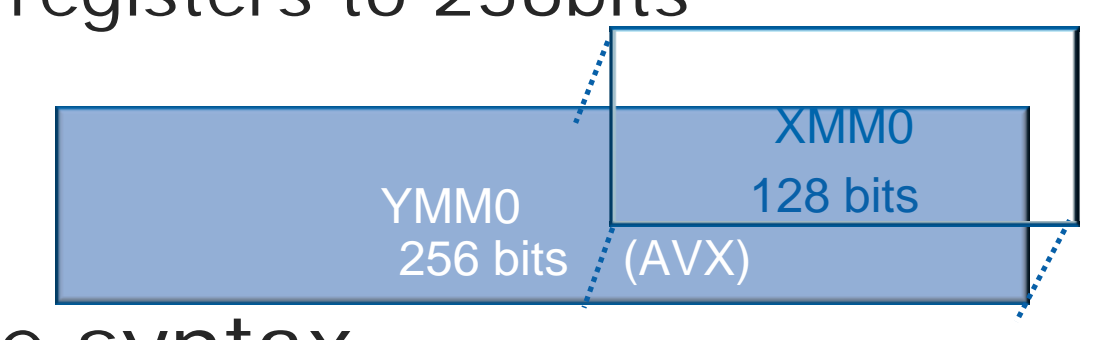

- VADDPS ymm1, ymm2, ymm3

- New Operations to enhance vectorization

- Broadcasts

- Masked load \& store 


\section{Intel ${ }^{\circledR}$ Advanced Vector Extensions ( I ntel ${ }^{\circledR}$ AVX)}

- Doubling the FLOPs in a cool manner

- Extend SSE FP instruction set to 256 bits operand size

- Intel AVX extends all 16 XMM registers to 256bits

- New, non-destructive source syntax

- VADDPS ymm1, ymm2, ymm3

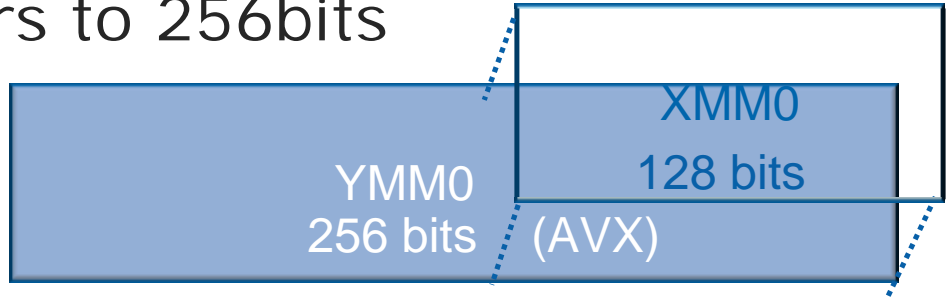

- New Operations to enhance vectorization

- Broadcasts

- Masked load \& store

\section{Intel ${ }^{\circledR}$ AVX is a "Cool" Architecture}

Vectors are a natural data-type for many applications

Wider vectors and non-destructive source specify more work with fewer instructions

Extending the existing state is area and power efficient 


\section{Execution Cluster - A Look Inside}

Scheduler sees matrix:

-3 "ports" to 3

"stacks" of

execution units

- General Purpose Integer

$$
\begin{array}{ll}
\text { - } & \text { SIMD (Vector) } \\
& \text { Integer } \\
\text { - SIMD Floating } & \text { Soint }
\end{array}
$$

-The challenge is to double the output of one of these stacks in a manner that is invisible to the others

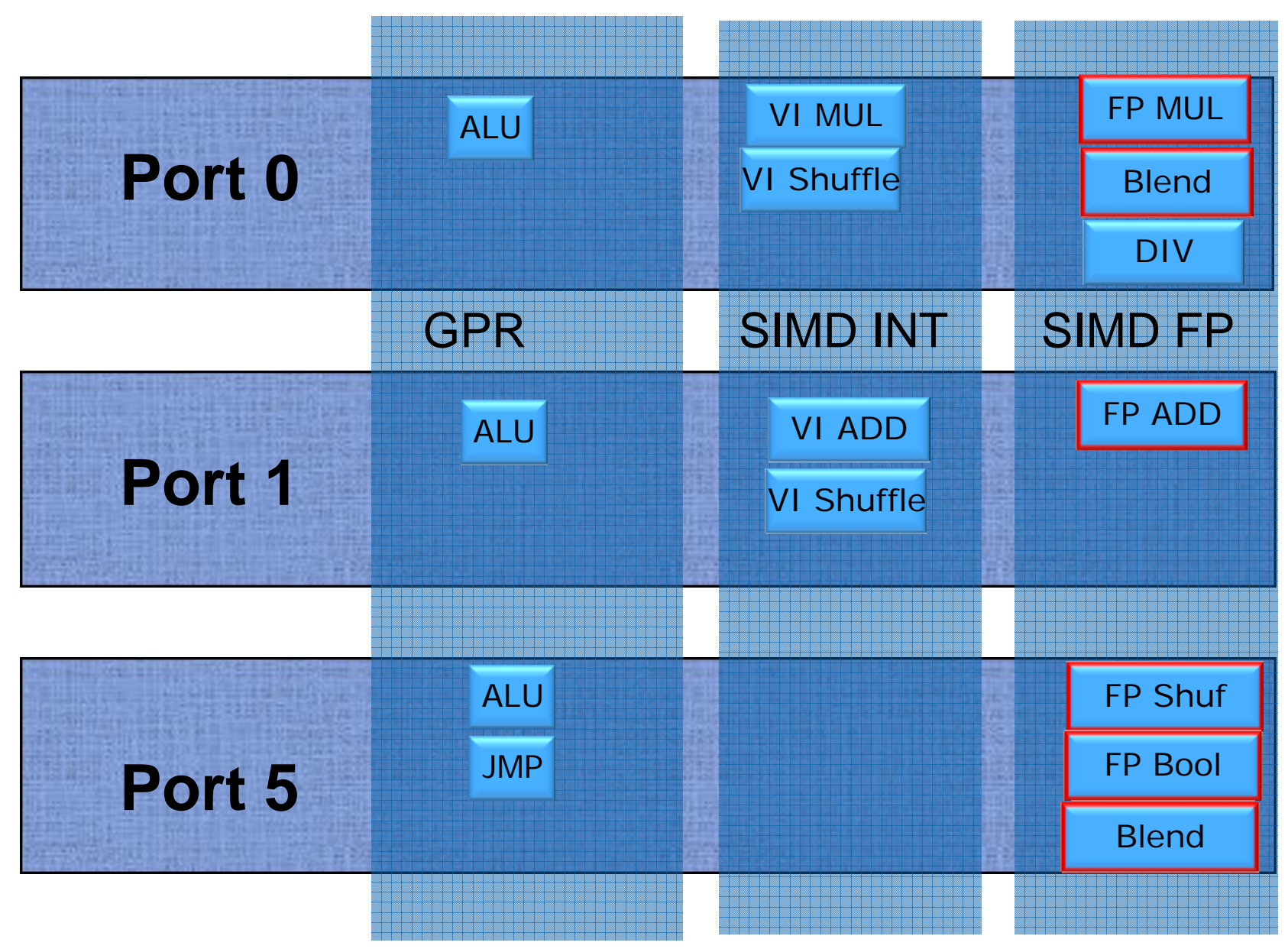




\section{Execution Cluster}

\section{Solution:}

- Repurpose existing datapaths to dual-use

- SIMD integer and legacy SIMD FP use legacy stack style

- Intel ${ }^{\circledR} \mathrm{AVX}$ utilizes both 128-bit execution stacks

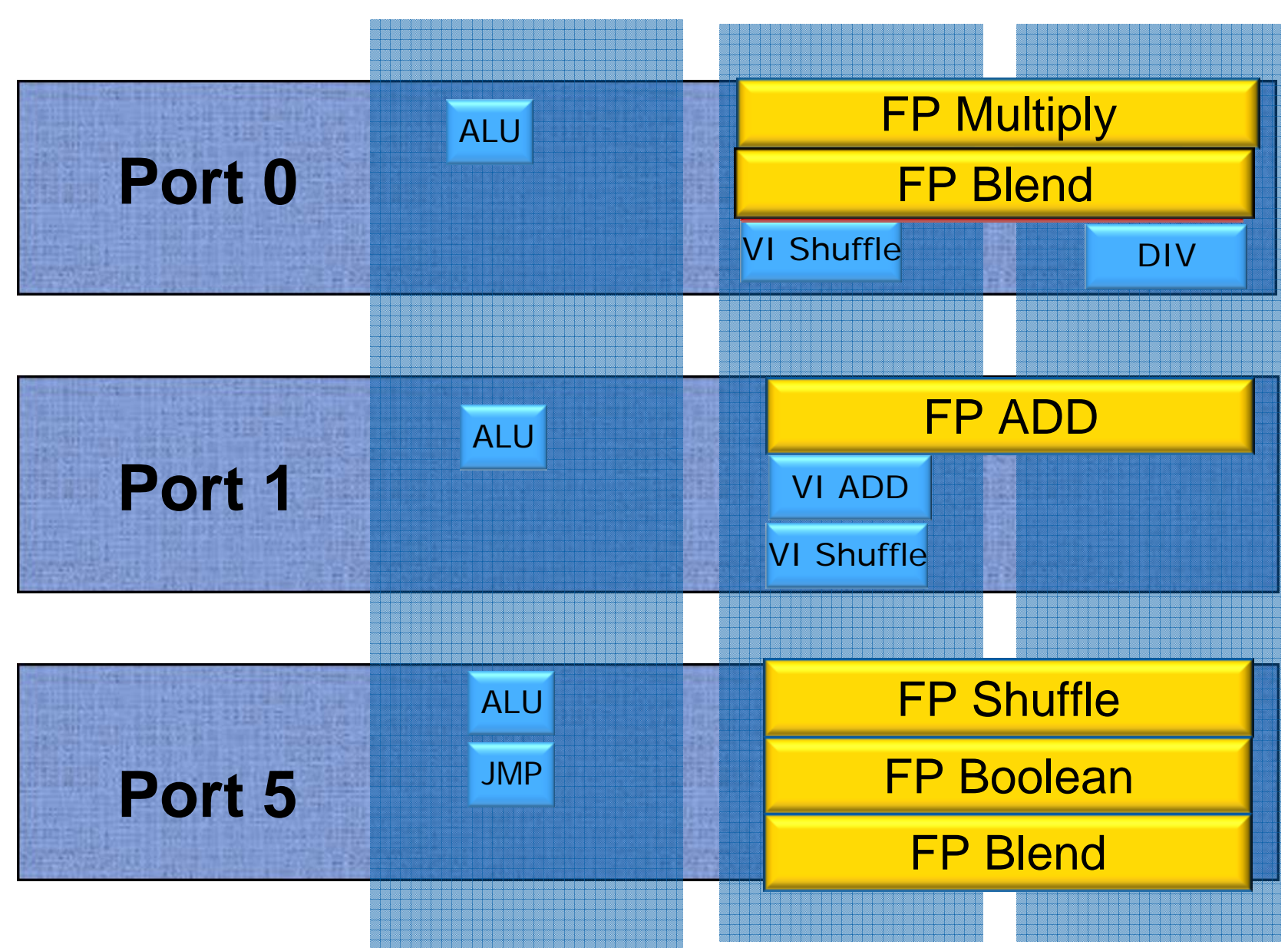




\section{Execution Cluster}

\section{Solution:}

- Repurpose existing datapaths to dual-use

- SIMD integer and legacy SIMD FP use legacy stack style

- Intel ${ }^{\circledR} \mathrm{AVX}$ utilizes both 128-bit execution stacks

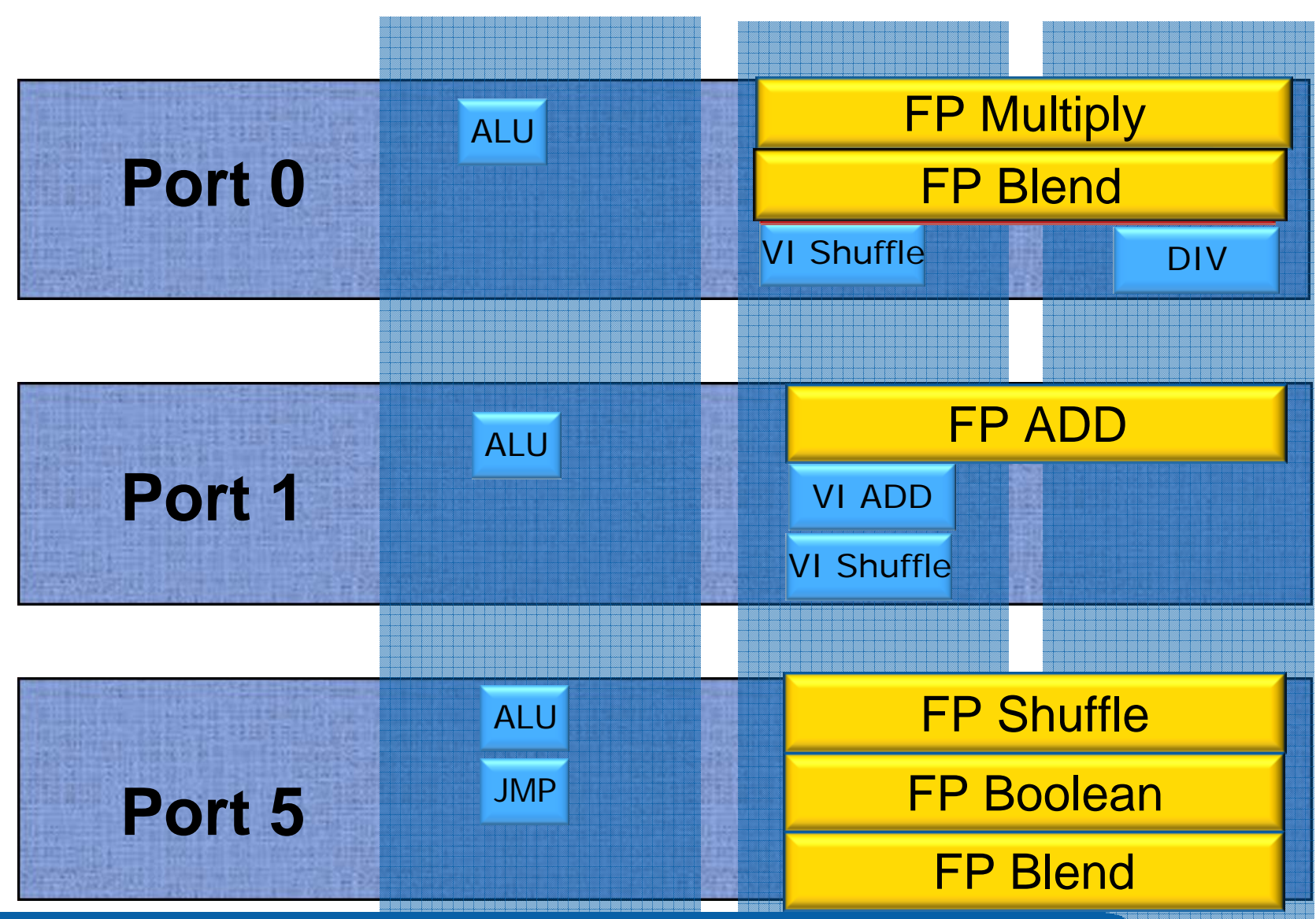

"Cool" I mplementation of I ntel AVX

256-bit Multiply + 256-bit ADD + 256-bit Load per clock... Double your FLOPs with great energy efficiency 


\section{Memory Cluster}

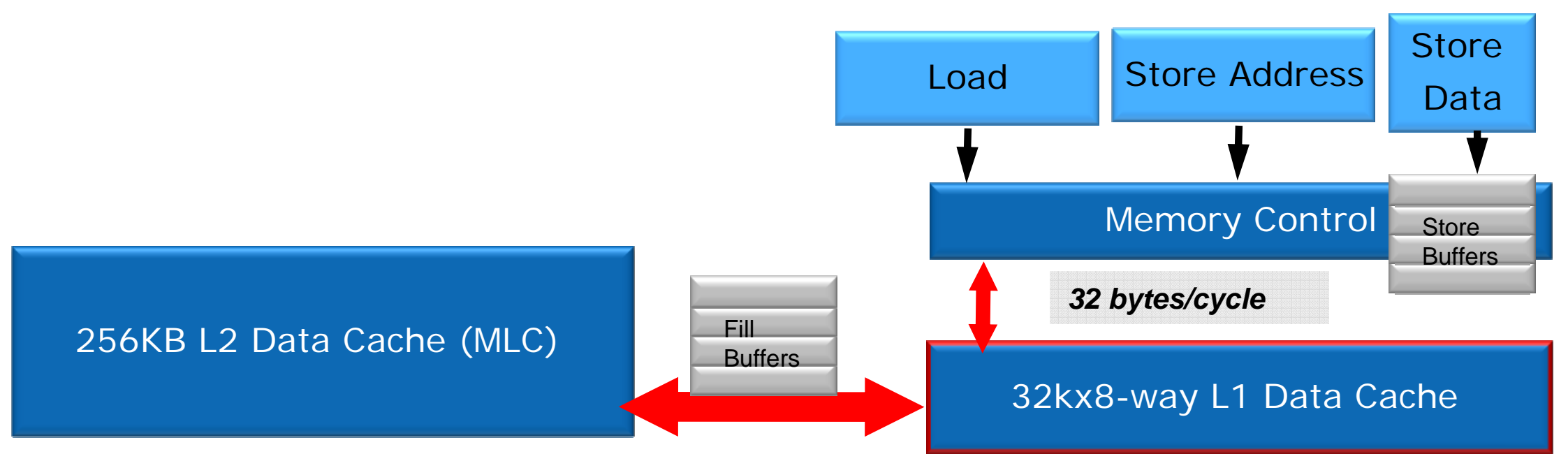

- Memory Unit can service two memory requests per cycle

- 16 bytes load and 16 bytes store per cycle

\section{Challenge to the Memory Cluster Architects}

Maintain the historic bytes/flop ratio of SSE for Intel ${ }^{\circledR} \mathrm{AVX}$

... and do so in a "cool" manner 


\section{Memory Cluster in Sandy Bridge}

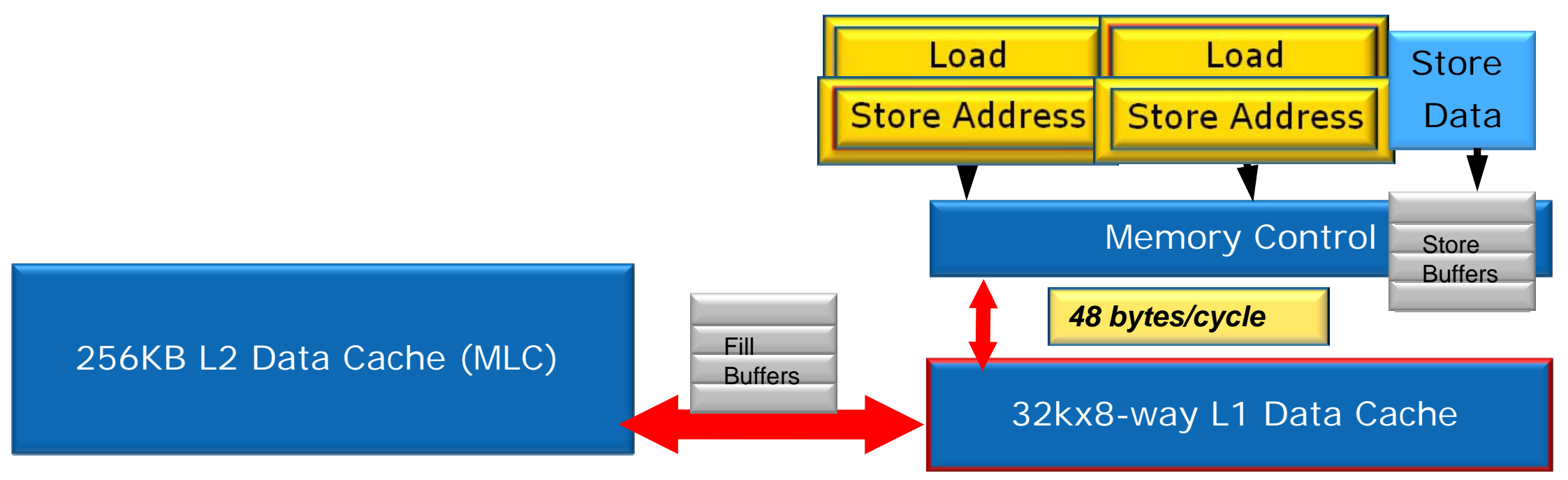

- Solution : Dual-Use the existing connections

- Make load/store pipes symmetric

- Memory Unit services three data accesses per cycle

- 2 read requests of up to 16 bytes AND 1 store of up to 16 bytes

- Internal sequencer deals with queued requests 


\section{Memory Cluster in Sandy Bridge}

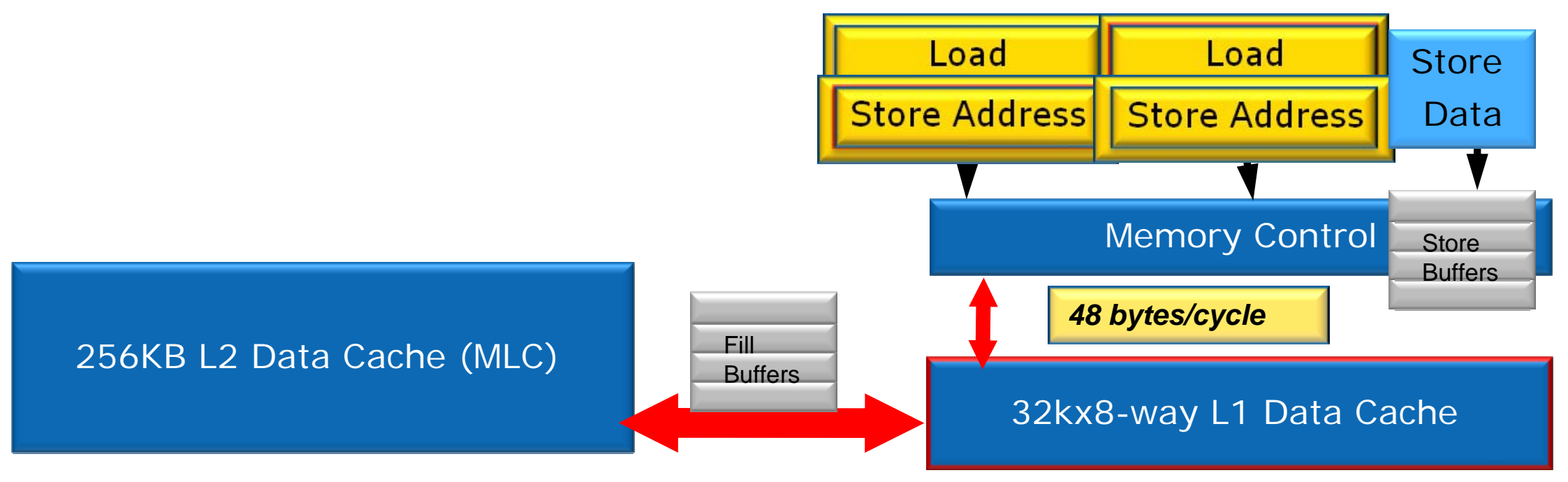

- Solution : Dual-Use the existing connections

- Make load/store pipes symmetric

- Memory Unit services three data accesses per cycle

- 2 read requests of up to 16 bytes AND 1 store of up to 16 bytes

- Internal sequencer deals with queued requests

Second Load Port is one of highest performance features Required to keep Intel ${ }^{\circledR}$ Advanced Vector Extensions (Intel ${ }^{\circledR}$ AVX) Instruction Set fed linear power/performance means its "Cool" 


\section{Putting it together Sandy Bridge Microarchitecture}

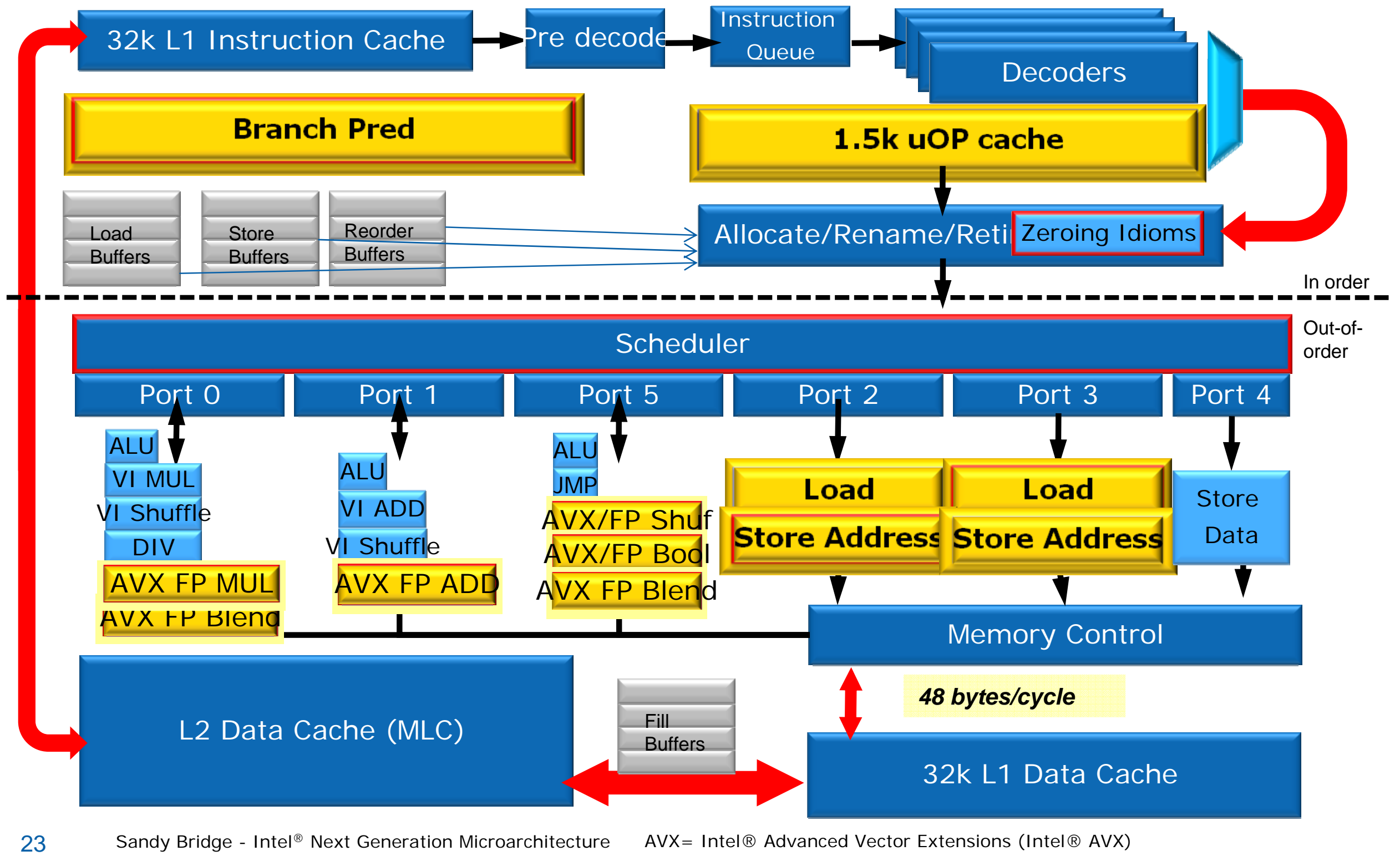




\section{Other Architectural Extensions}

- Cryptography Instruction Throughput Enhancements

- throughput for AES instructions introduced in Westmere

- Large Number Arithmetic Throughput Enhancements

- ADC (Add with Carry) throughput doubled

- Multiply (64-bit multiplicands with 128-bit product)

- $25 \%$ speedup on existing RSA binaries!

- State Save/Restore Enhancements

- New state added in Intel ${ }^{\circledR}$ Advanced Vector Extensions (Intel ${ }^{\circledR}$ AVX)

- HW monitors features used by applications

- Only saves/restores state that is used 


\section{Sandy Bridge Processor Core Summary}

- Build upon the successful Nehalem processor core

- Converged building block for mobile, desktop, and server

- "Cool" and "Really Cool" features

- Improve performance/power and performance/area

- Extends the architecture for important new applications

- Floating Point and Throughput Applications

- Intel ${ }^{\circledR}$ Advanced Vector Extensions (Intel ${ }^{\circledR}$ AVX) - Significant boost for selected compute intensive apps

- Security

- AES (Advanced Encryption Standard) Instructions speedup

- Large Integer RSA and SHA speedups

- OS/VMM and server related features

- State save/ restore optimizations 


\section{Sandy Bridge}

Microarchitecture

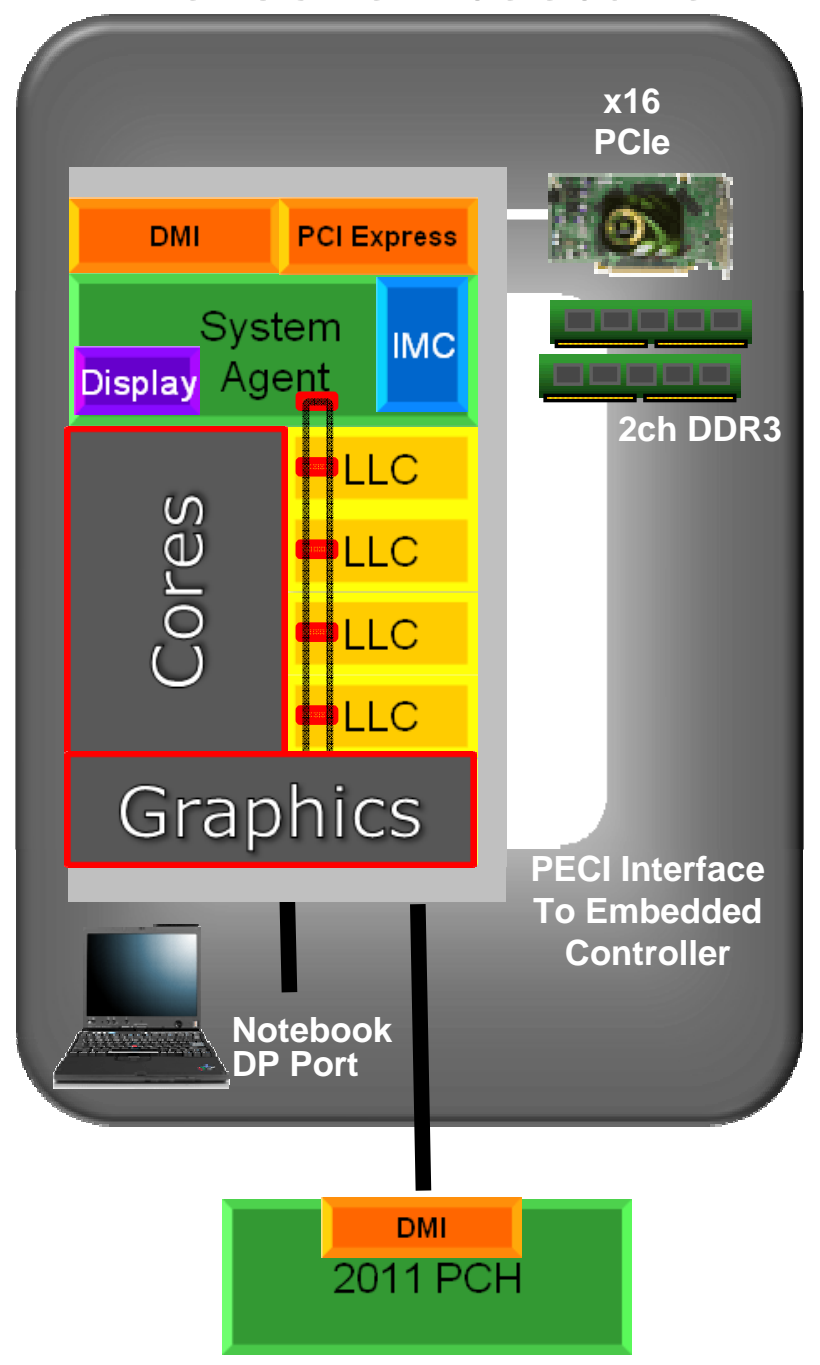

\section{System Agent, Ring Architecture and Other I nnovations}




\section{I ntegration: Optimization Opportunities}

- Dynamically redistribute power between Cores \& Graphics

- Tight power management control of all components, providing better granularity and deeper idle/sleep states

- Three separate power/ frequency domains: System Agent (Fixed), Cores+Ring, Graphics (Variable)

- High BW Last Level Cache, shared among Cores and Graphics

- Significant performance boost, saves memory bandwidth and power

- Integrated Memory Controller and PCI Express* ports

- Tightly integrated with Core/Graphics/LLC domain

- Provides low latency \& low power - remove intermediate busses

- Bandwidth is balanced across the whole machine, from Core/Graphics all the way to Memory Controller

- Modular uArch for optimal cost/ power/ performance

- Derivative products done with minimal effort/time 


\section{Scalable Ring On-die I nterconnect}

- Ring-based interconnect between Cores, Graphics, Last Level Cache (LLC) and System Agent domain

- Composed of 4 rings

- 32 Byte Data ring, Request ring, Acknowledge ring and Snoop ring

- Fully pipelined at core frequency/voltage: bandwidth, latency and power scale with cores

- Massive ring wire routing runs over the LLC with no area impact

- Access on ring always picks the shortest path - minimize latency

- Distributed arbitration, sophisticated ring protocol to handle coherency, ordering, and core interface

- Scalable to servers with large number of processors

\section{High Bandwidth, Low Latency, Modular}




\section{Cache Box}

- Interface block

- Between Core/ Graphics/ Media and the Ring

- Between Cache controller and the Ring

- Implements the ring logic, arbitration, cache controller

- Communicates with System Agent for LLC misses, external snoops, non-cacheable accesses

- Full cache pipeline in each cache box

- Physical Addresses are hashed at the source to prevent hot spots and increase bandwidth

- Maintains coherency and ordering for the addresses that are mapped to it

- LLC is fully inclusive with "Core Valid Bits" eliminates unnecessary snoops to cores

- Runs at core voltage/ frequency, scales with Cores

\section{Distributed coherency $\&$ ordering; Scalable Bandwidth, Latency $\&$ Power}




\section{Ring Illustration: Clean LLC Hit}

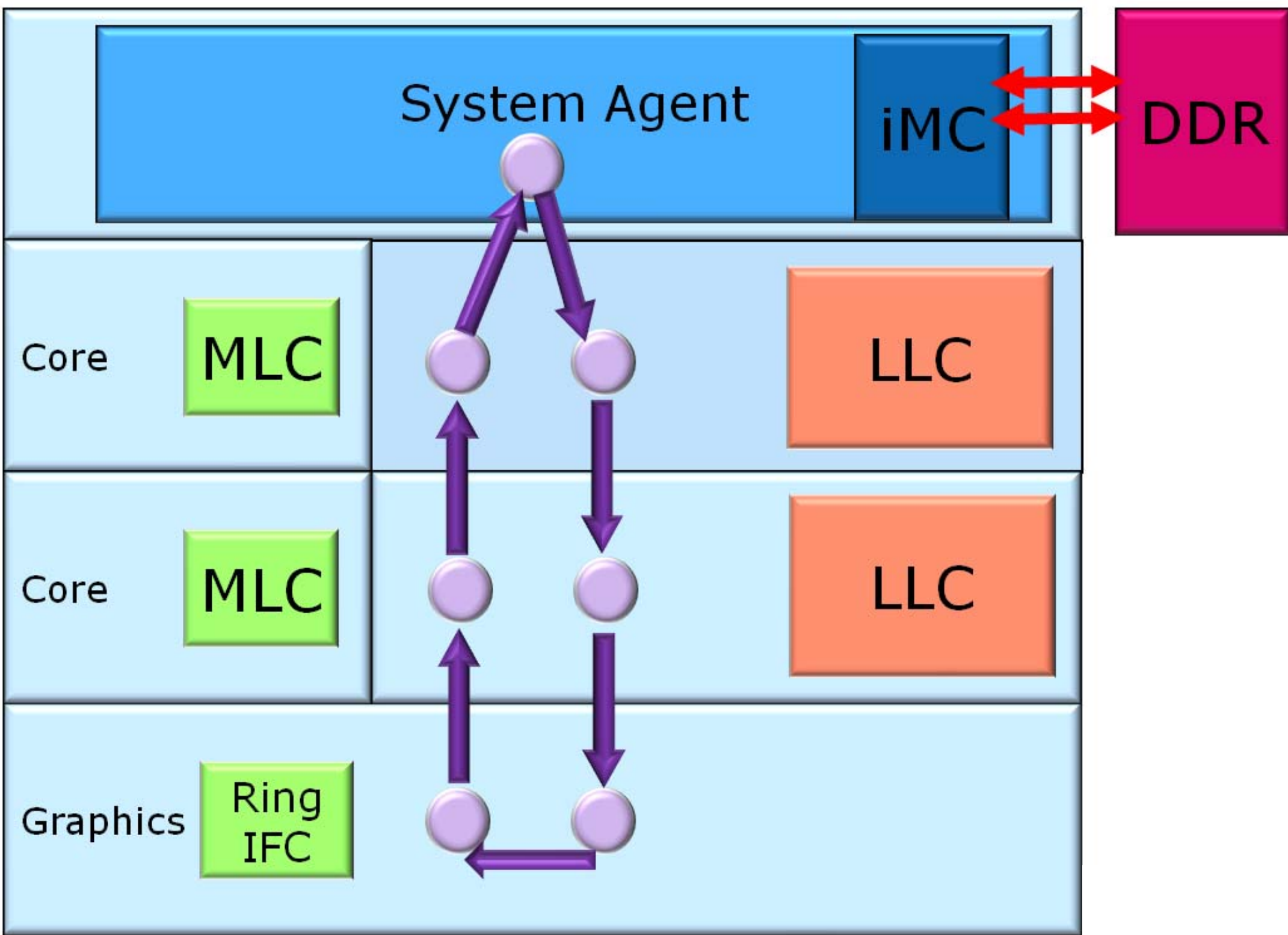




\section{Ring Illustration: Clean LLC Hit}

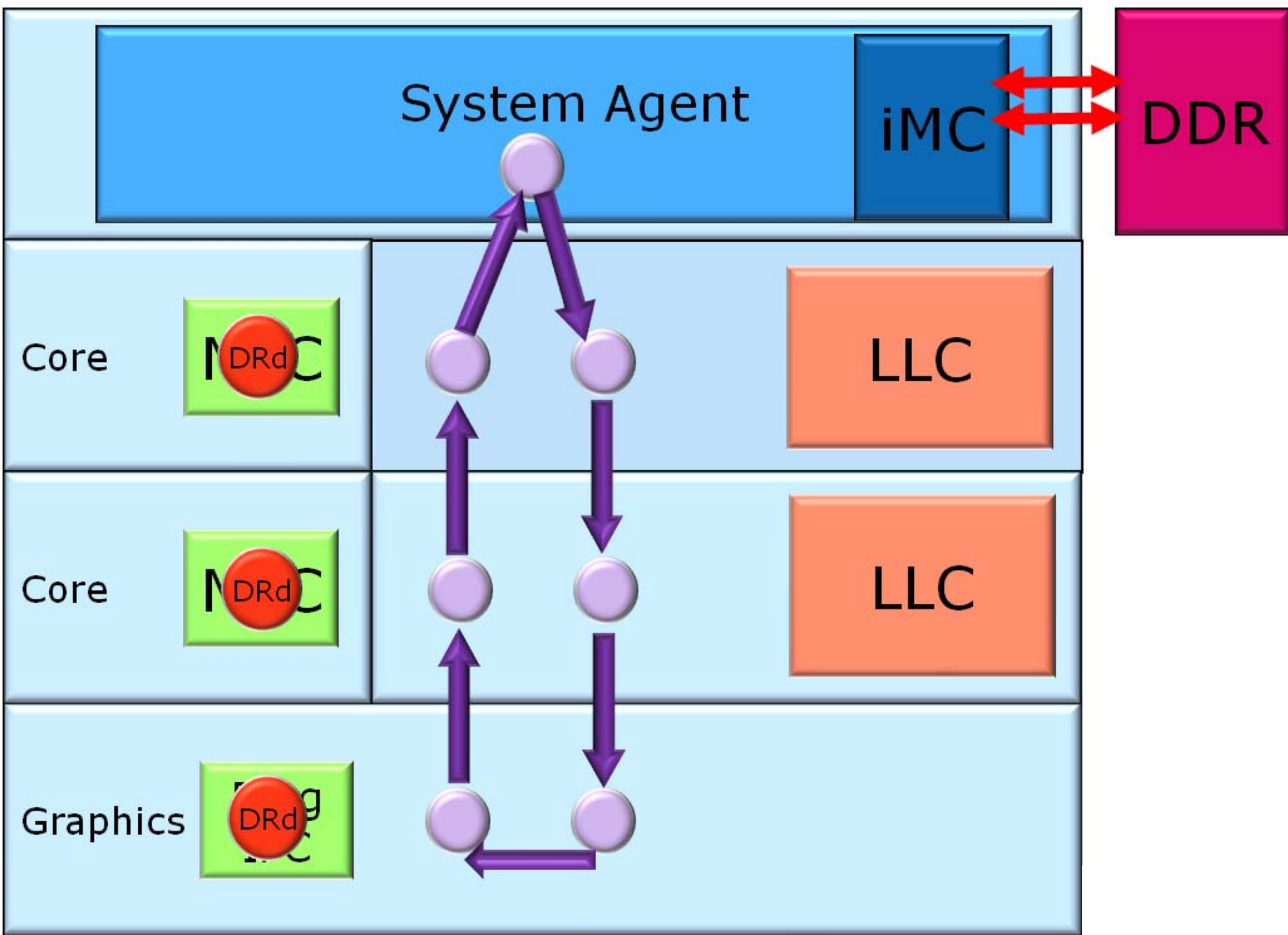




\section{Ring Illustration: Clean LLC Hit}

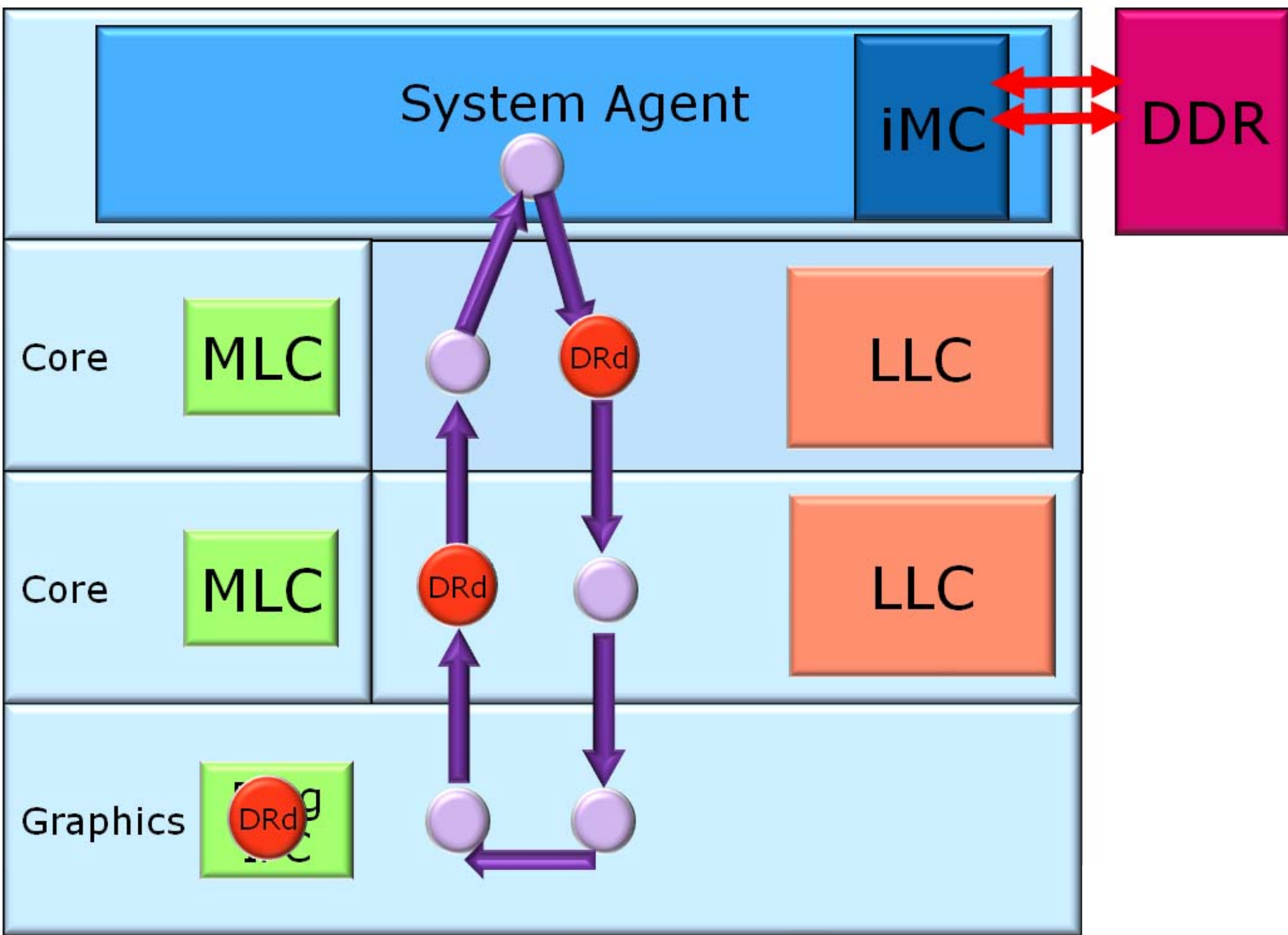




\section{Ring Illustration: Clean LLC Hit}

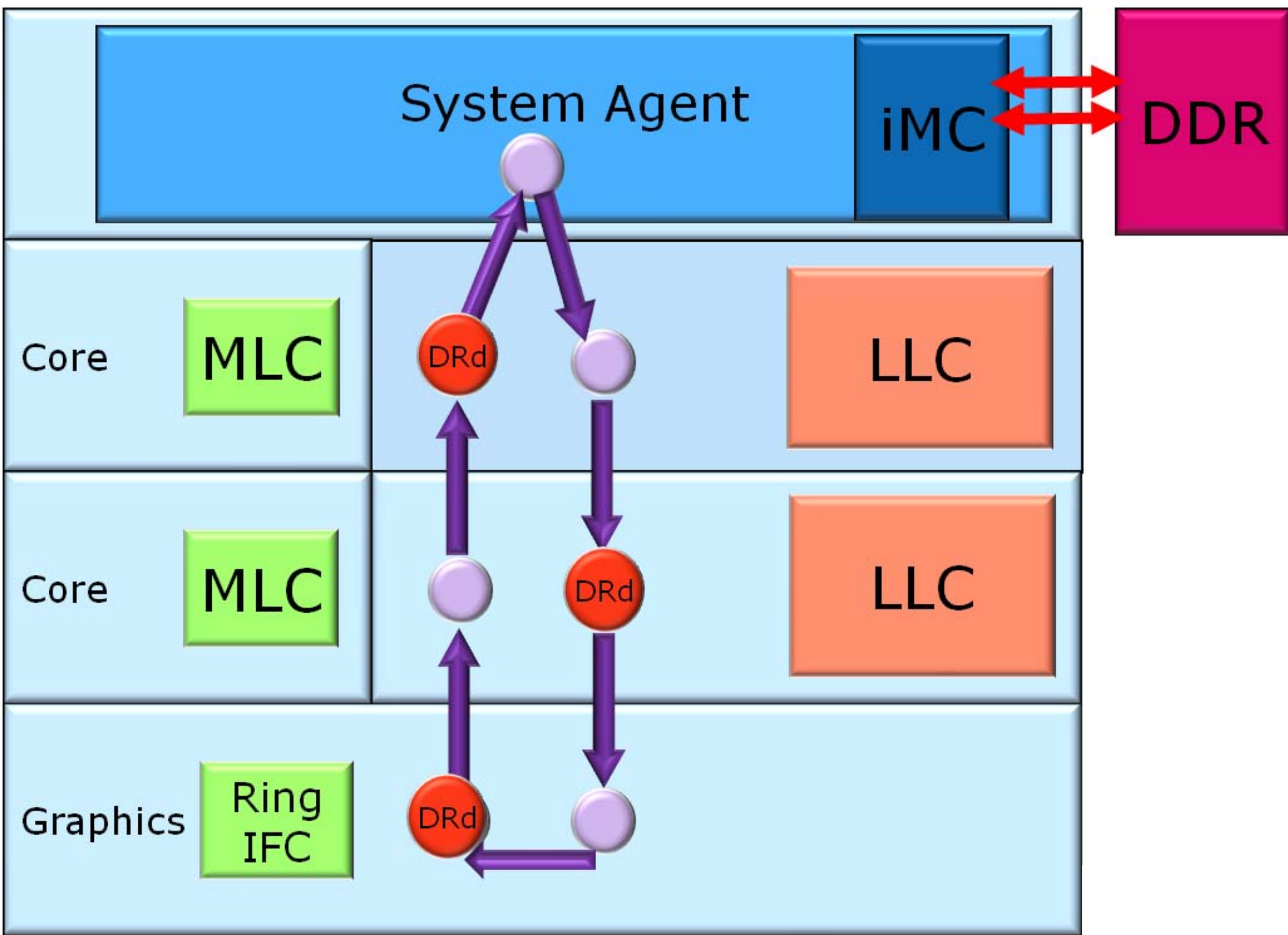




\section{Ring Illustration: Clean LLC Hit}

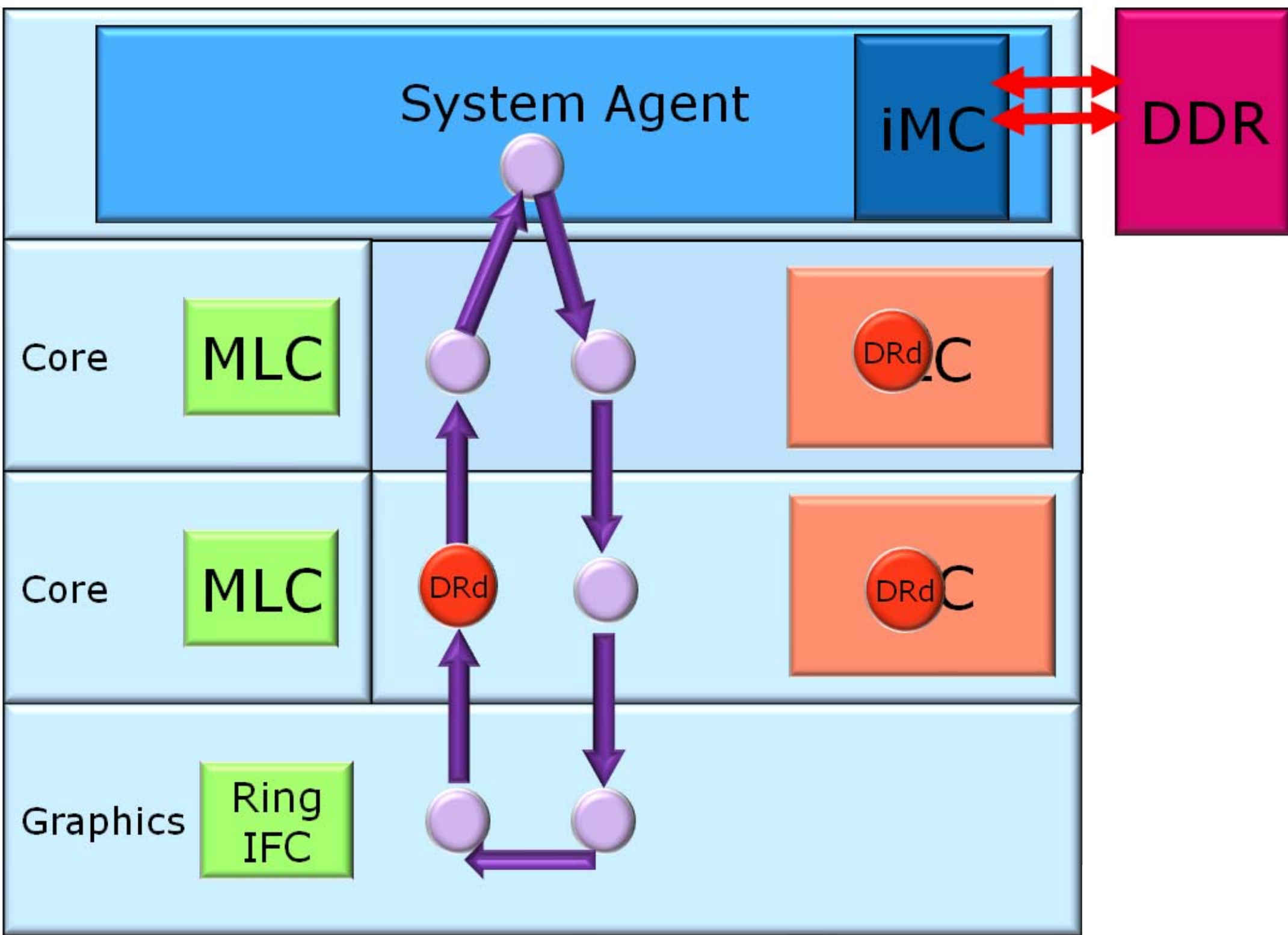




\section{Ring Illustration: Clean LLC Hit}

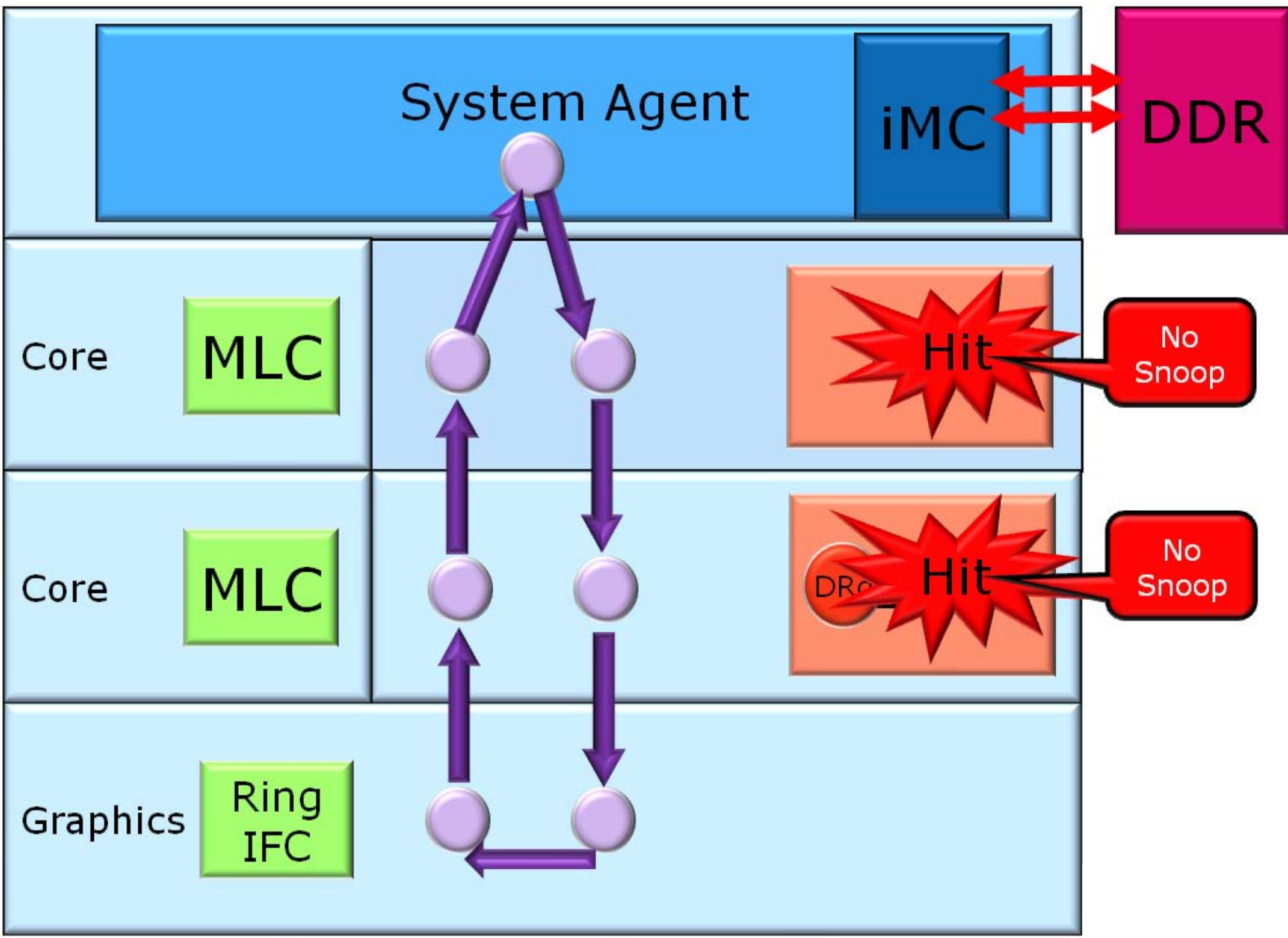




\section{Ring Illustration: Clean LLC Hit}

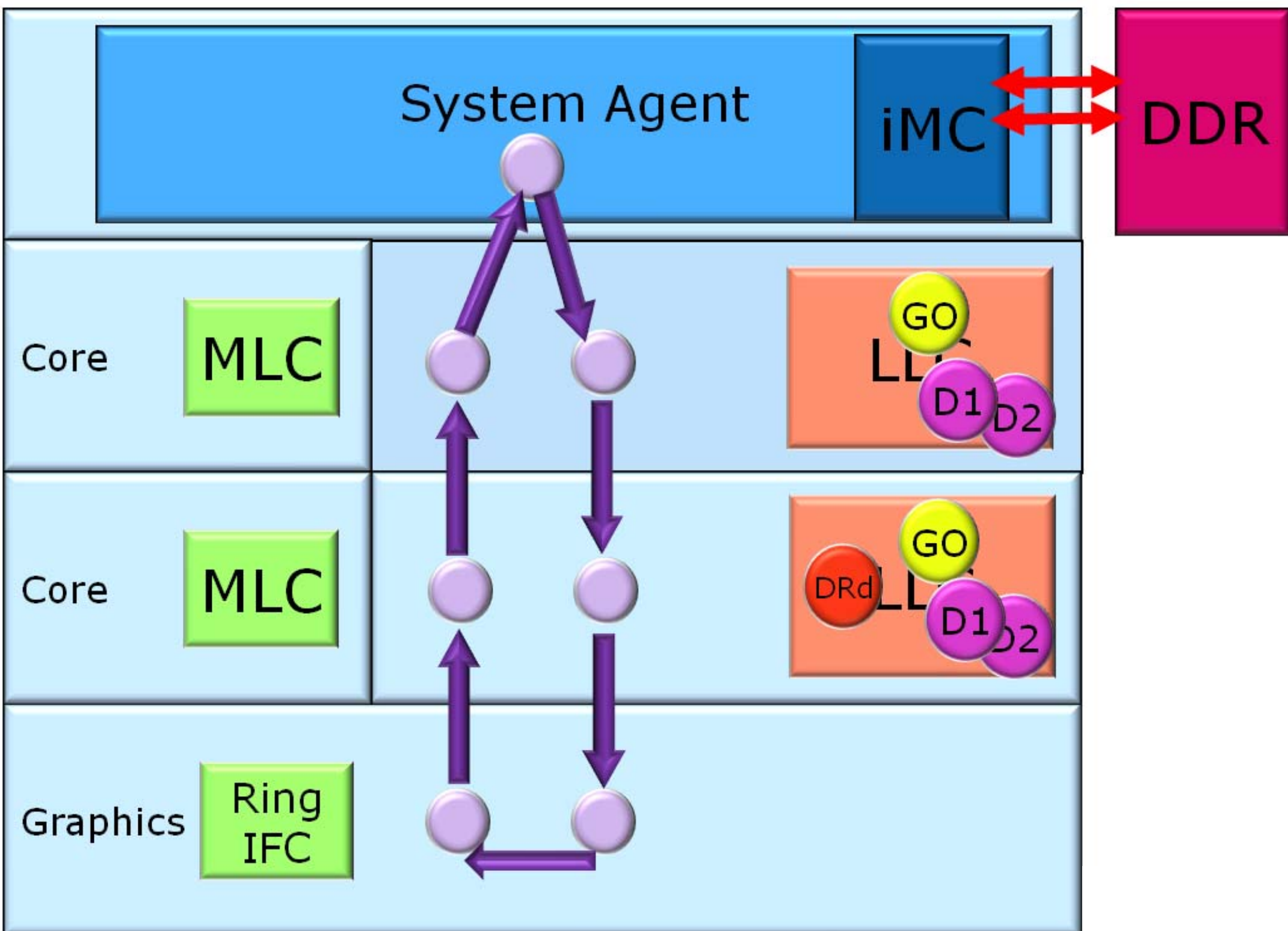




\section{Ring Illustration: Clean LLC Hit}

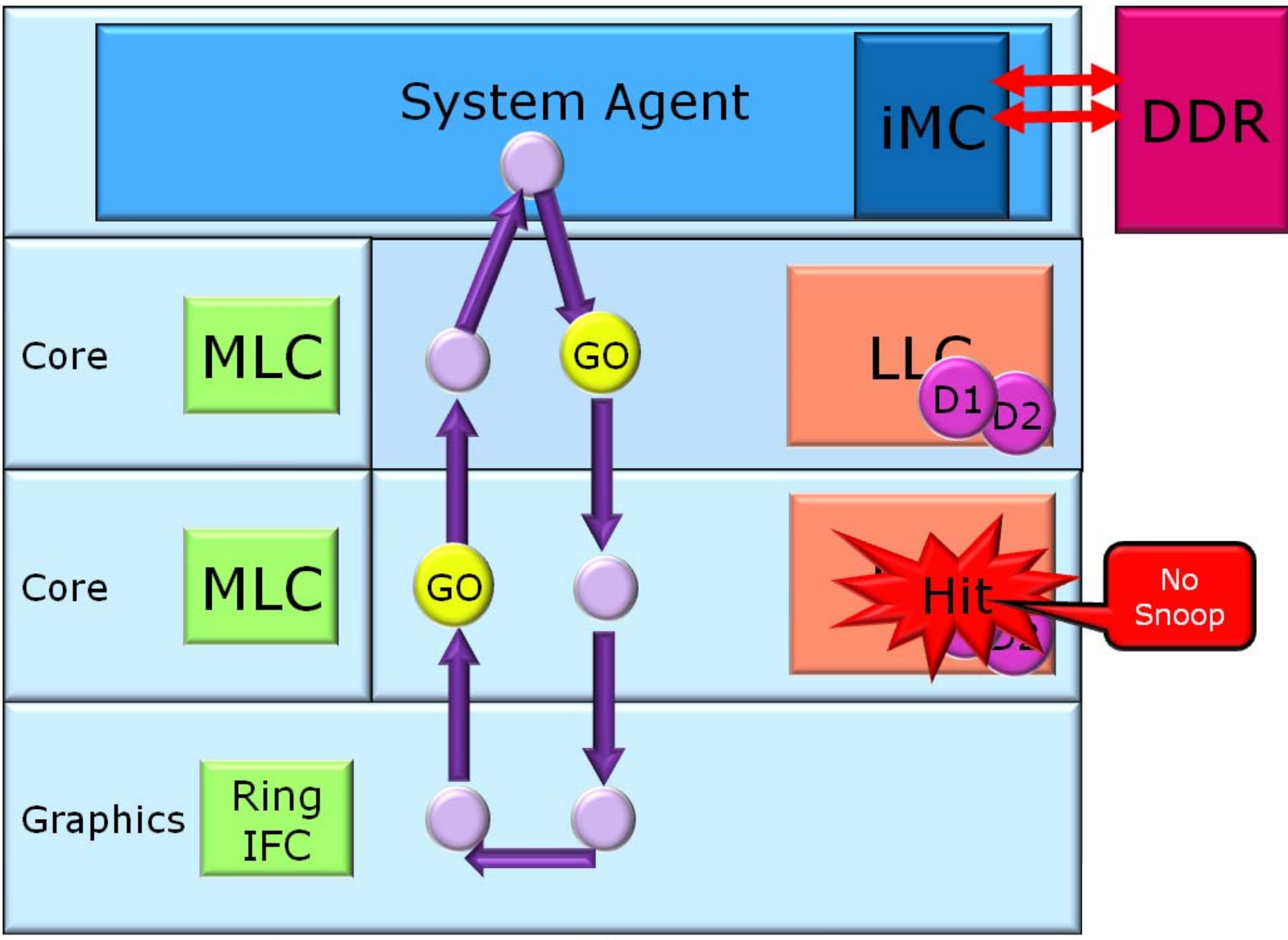




\section{Ring Illustration: Clean LLC Hit}

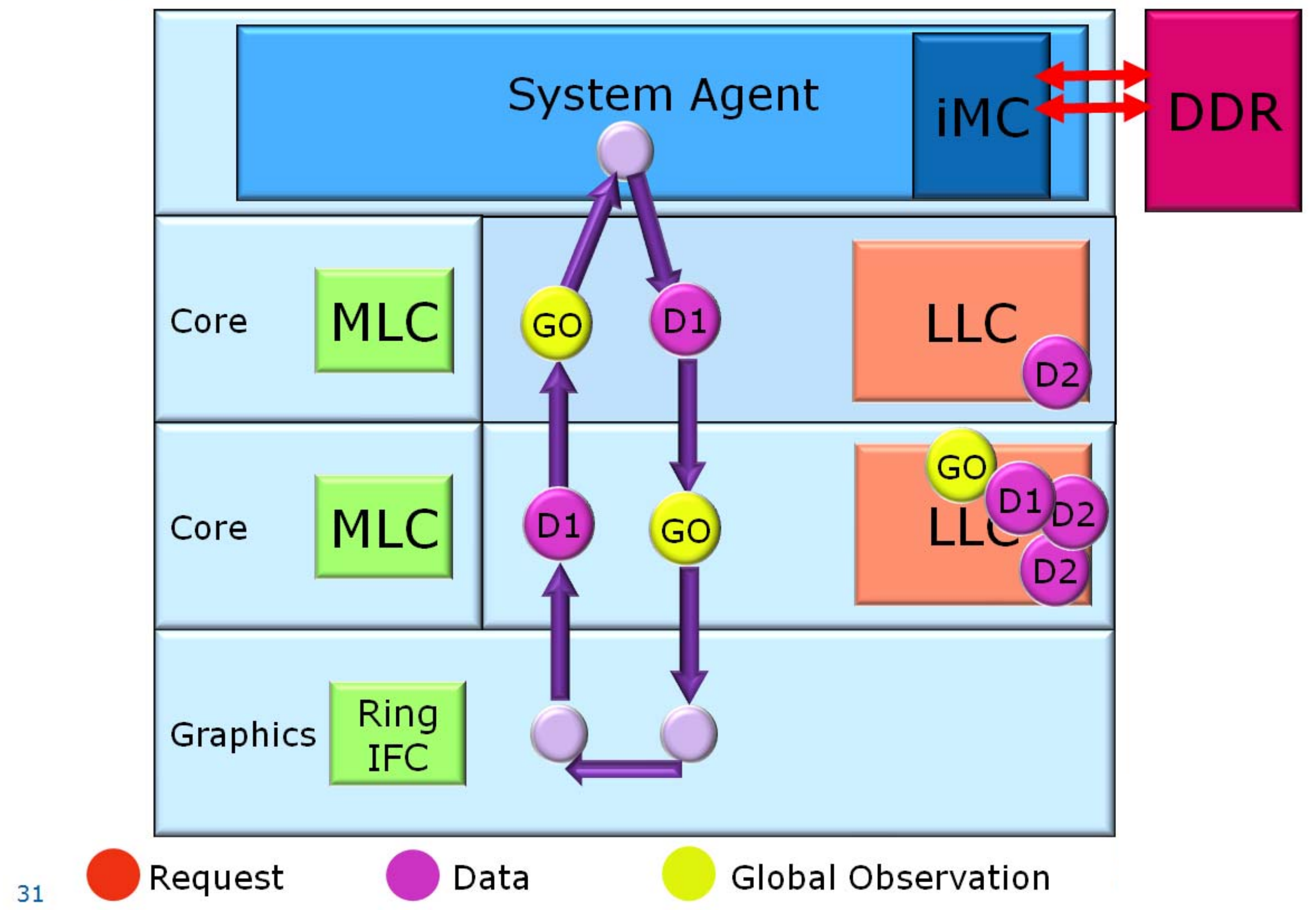




\section{Ring Illustration: Clean LLC Hit}

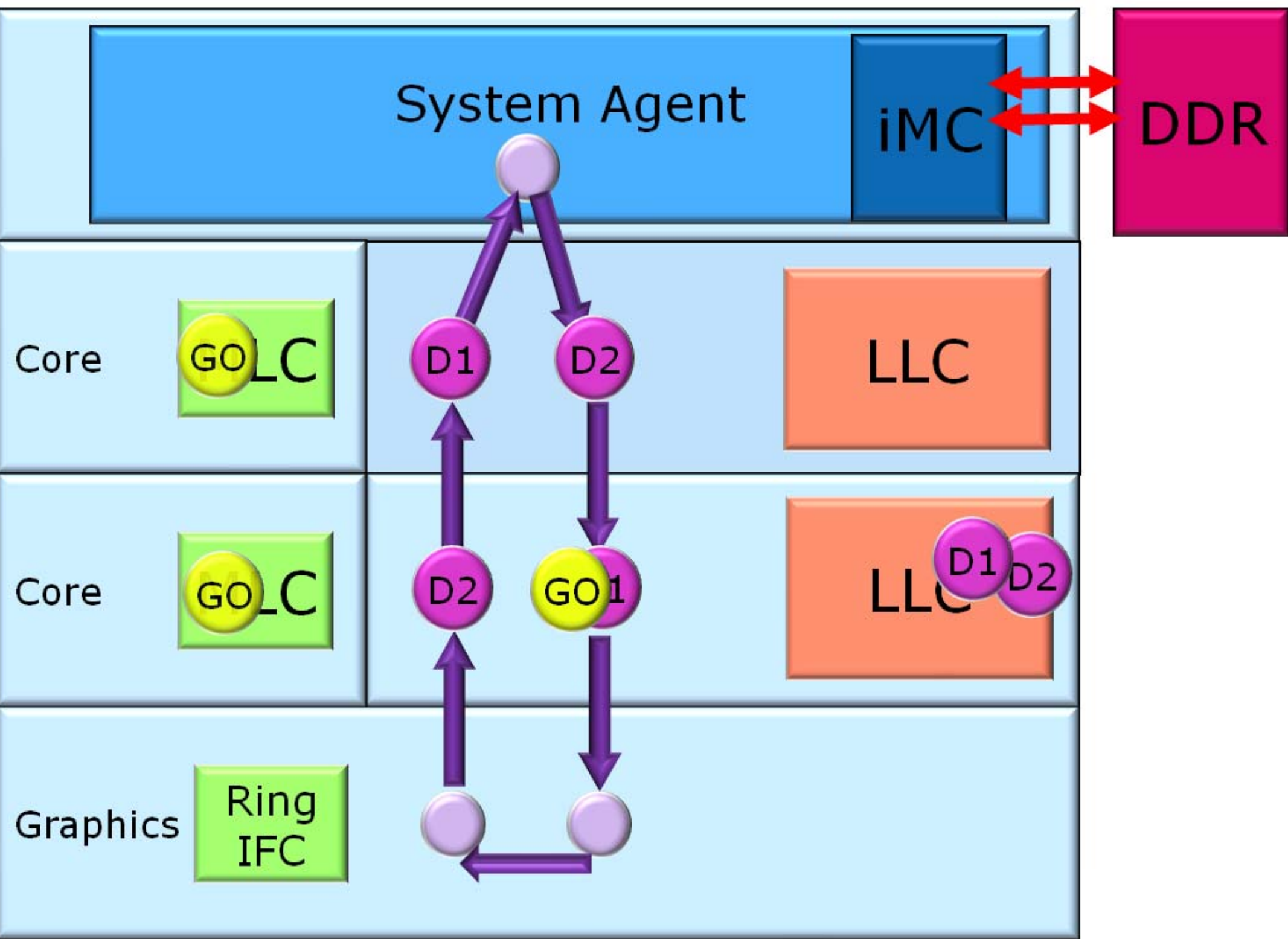




\section{Ring Illustration: Clean LLC Hit}

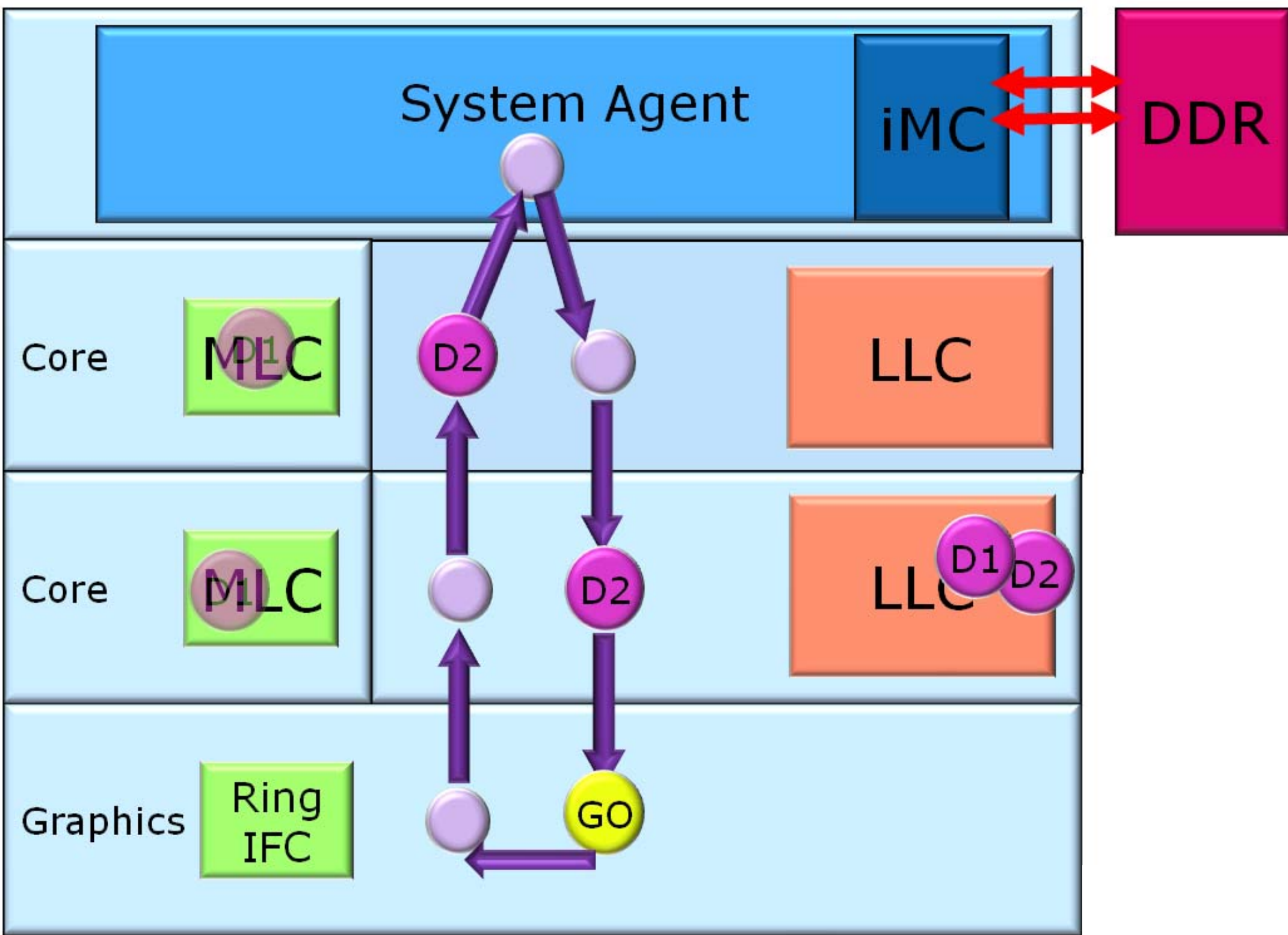




\section{Ring Illustration: Clean LLC Hit}

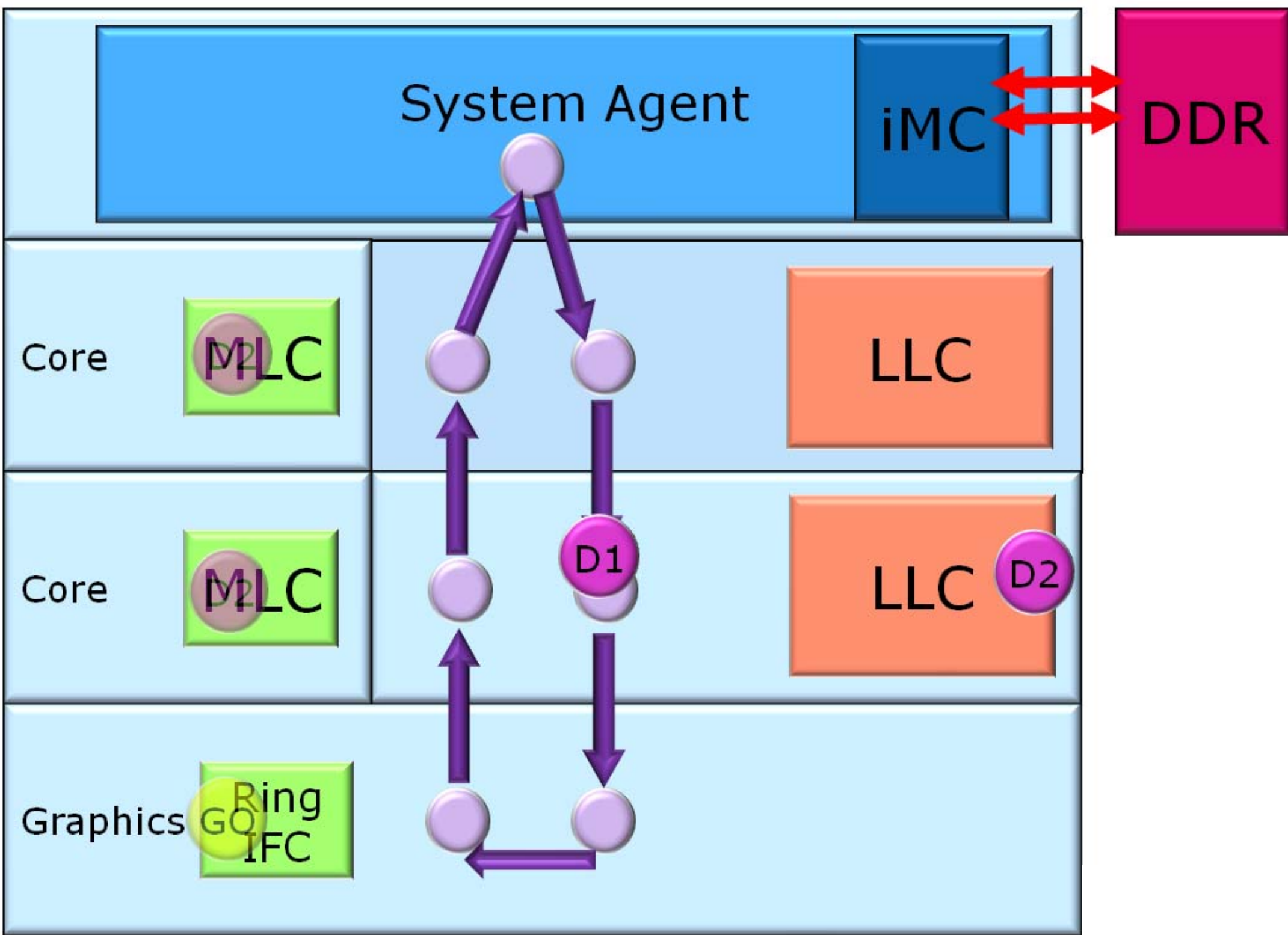




\section{Ring Illustration: Clean LLC Hit}

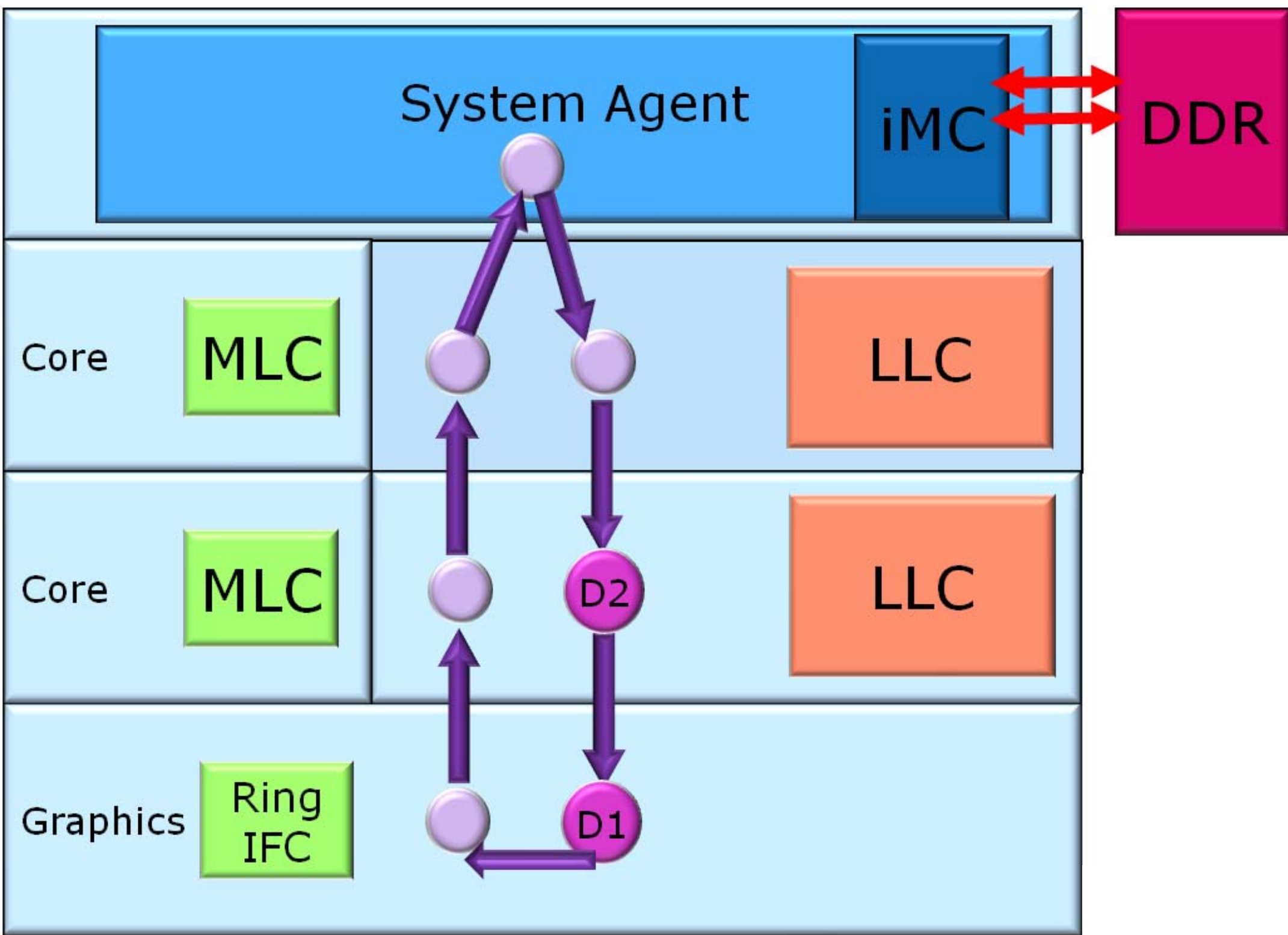




\section{Ring Illustration: Clean LLC Hit}

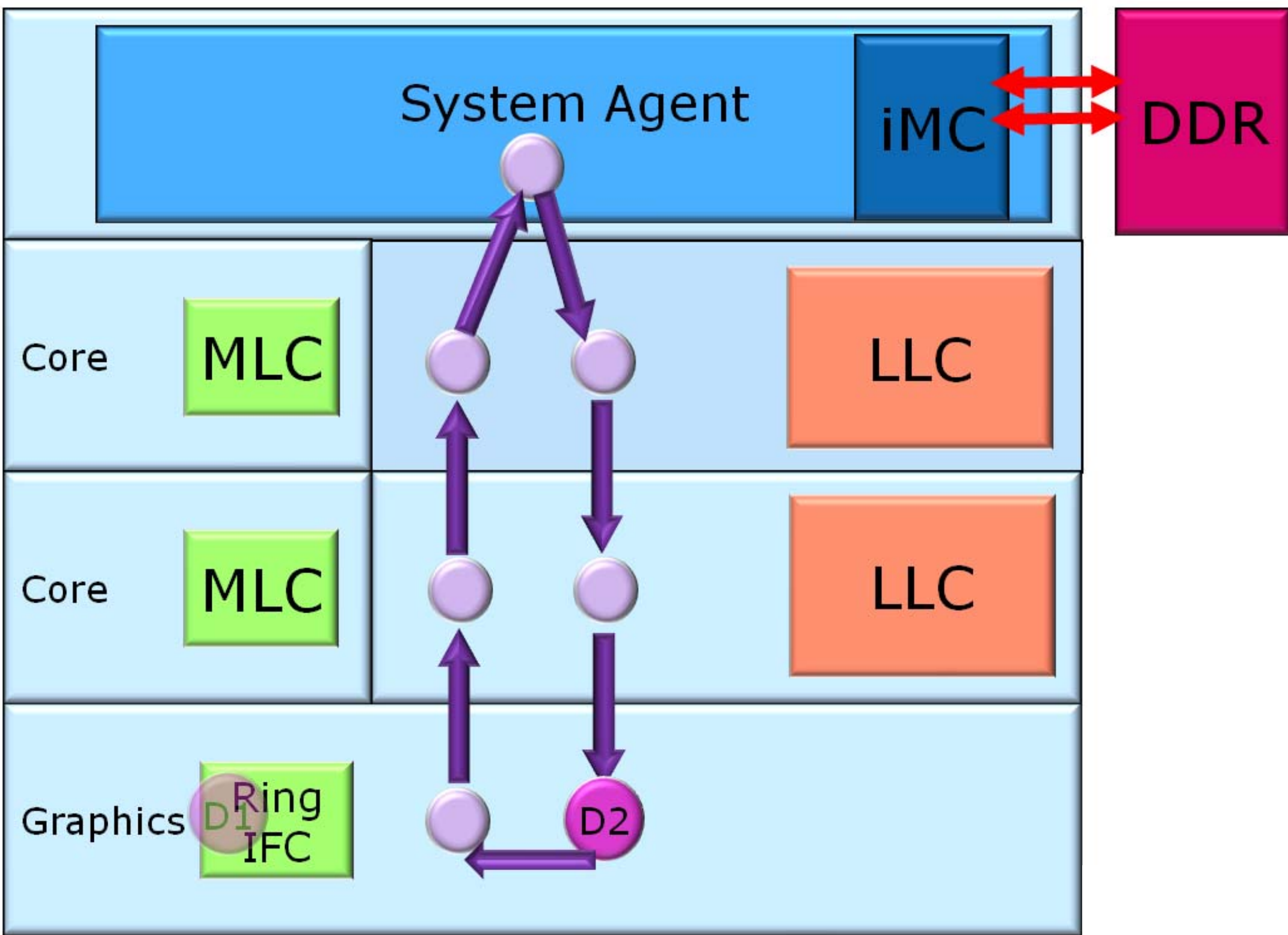




\section{Ring Illustration: Clean LLC Hit}

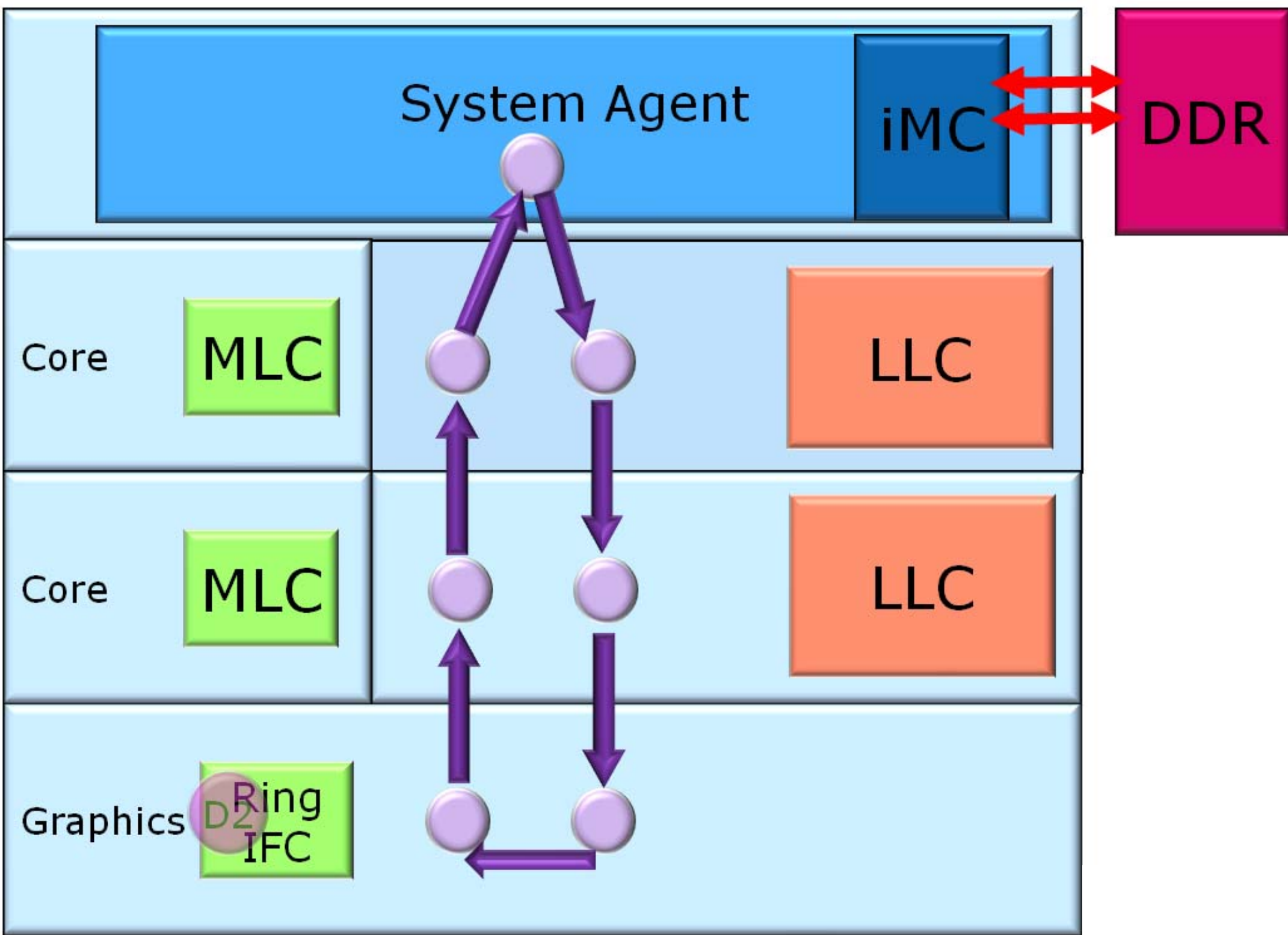




\section{Sandy Bridge LLC Sharing}

- LLC shared among all Cores, Graphics and Media

- Graphics driver controls which streams are cached/coherent

- Any agent can access all data in the LLC, independent of who allocated the line, after memory range checks

- Controlled LLC way allocation mechanism to prevent thrashing between Core/graphics

- Multiple coherency domains

- IA Domain (Fully coherent via cross-snoops)

- Graphic domain (Graphics virtual caches, flushed to IA domain by graphics engine)

- Non-Coherent domain (Display data, flushed to memory by graphics engine)

Much higher Graphics performance, DRAM power savings, more DRAM BW

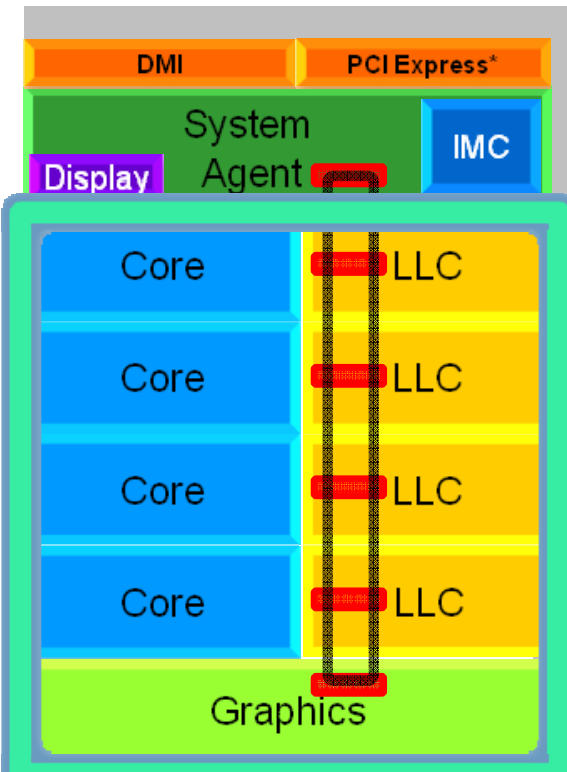

\section{available for Cores}




\section{Lean and Mean System Agent}

- Contains PCI Express*, DMI, Memory

Controller, Display Engine...

- Contains Power Control Unit

- Programmable uController, handles all power management and reset functions in the chip

- Smart integration with the ring

- Provides cores/Graphics / Media with high BW, Iow latency to DRAM/IO for best performance

- Handles IO-to-cache coherency

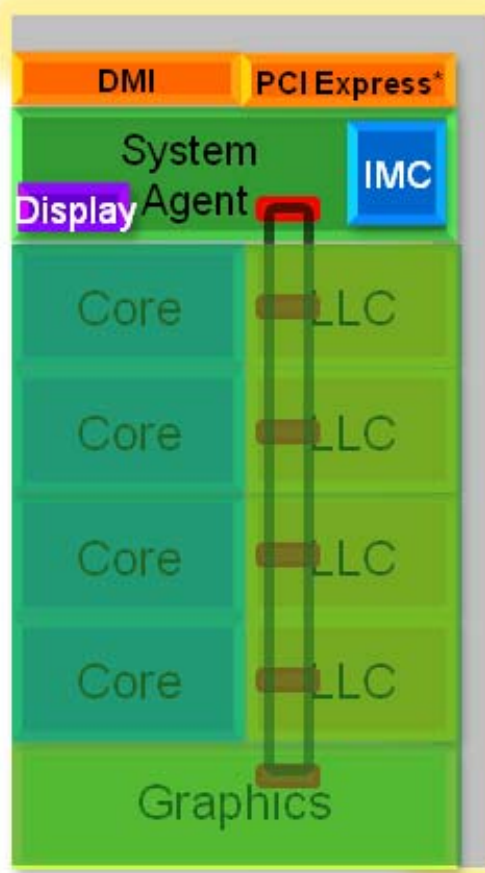




\section{Lean and Mean System Agent}

- Contains PCl Express*, DMI, Memory Controller, Display Engine...

- Contains Power Control Unit

- Programmable uController, handles all power management and reset functions in the chip

- Smart integration with the ring

- Provides cores/Graphics / Media with high BW, low latency to DRAM/IO for best performance

- Handles IO-to-cache coherency

- Separate voltage and frequency from ring/cores, Display integration for better battery life
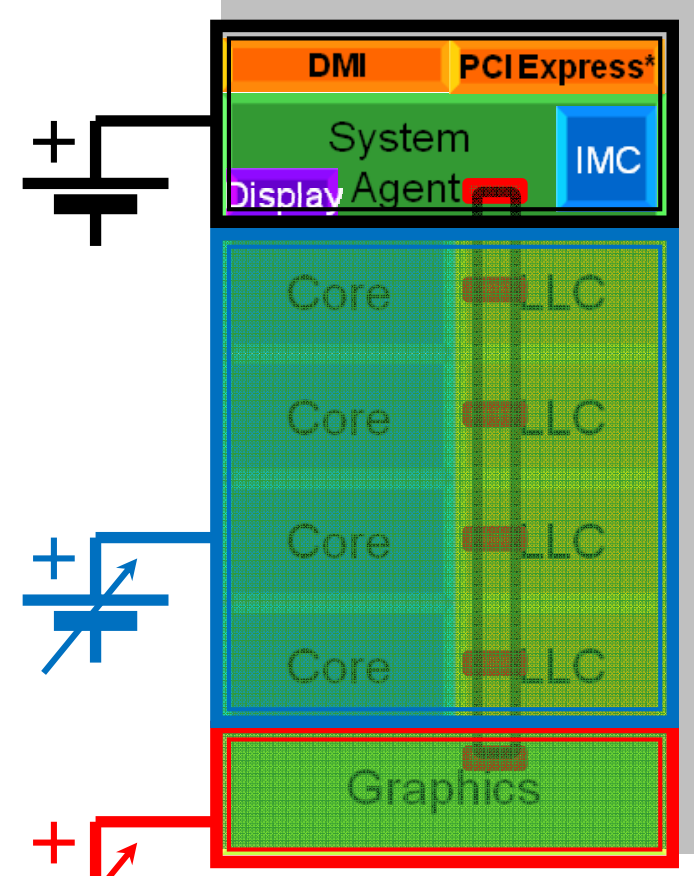

- Extensive power and thermal management for $\mathrm{PCl}$ Express* and DDR

\section{Smart I/ 0 I ntegration}


Power and Thermal Management 


\section{Usage Scenario: Responsive Behavior}

- Interactive work benefits from Next Generation Intel ${ }^{\circledR}$ Turbo Boost

- Idle periods intermixed with user actions

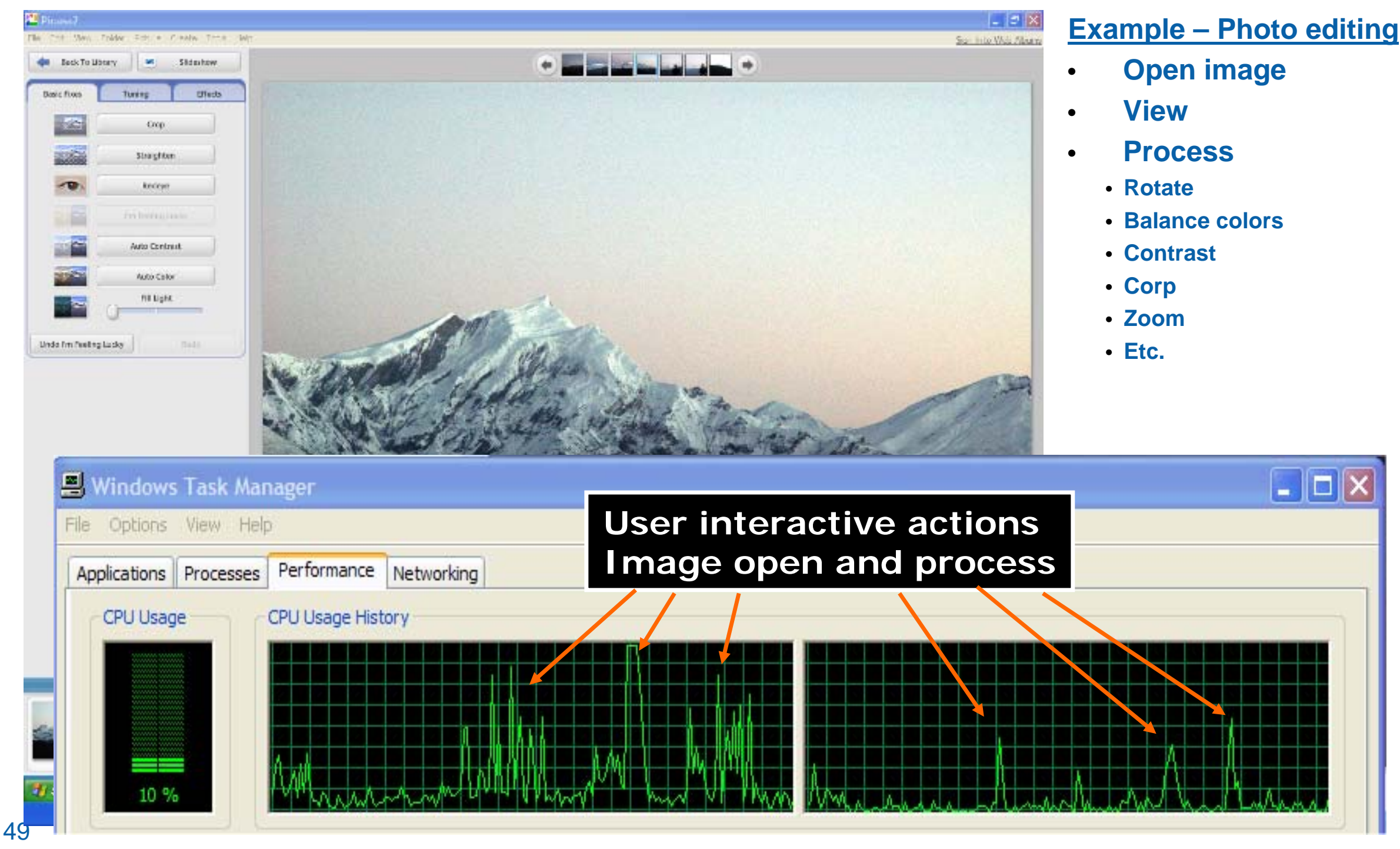




\section{I nnovative Concept: Thermal Capacitance}

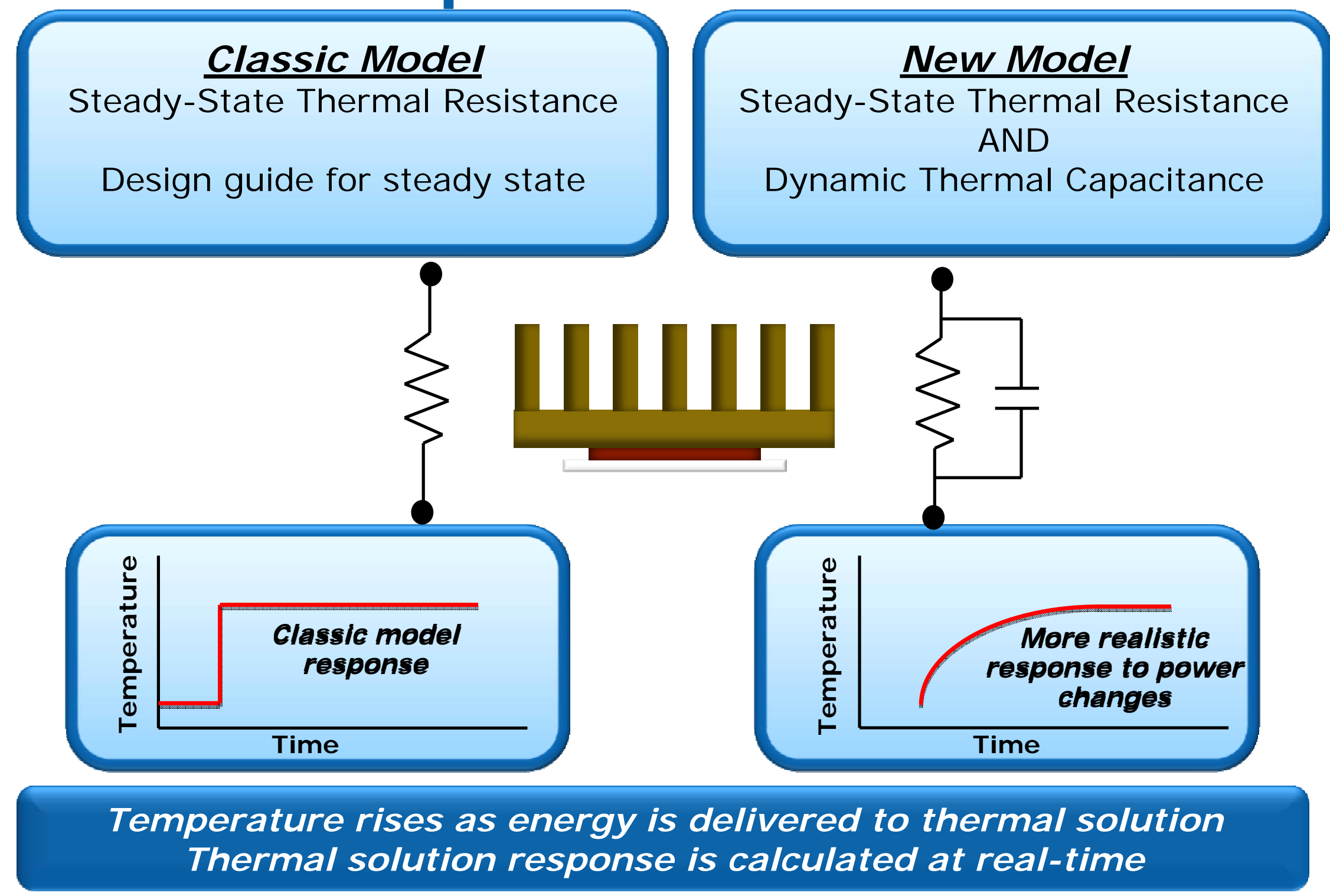




\section{Next Generation I ntel ${ }^{\circledR}$ Turbo Boost Benefit}

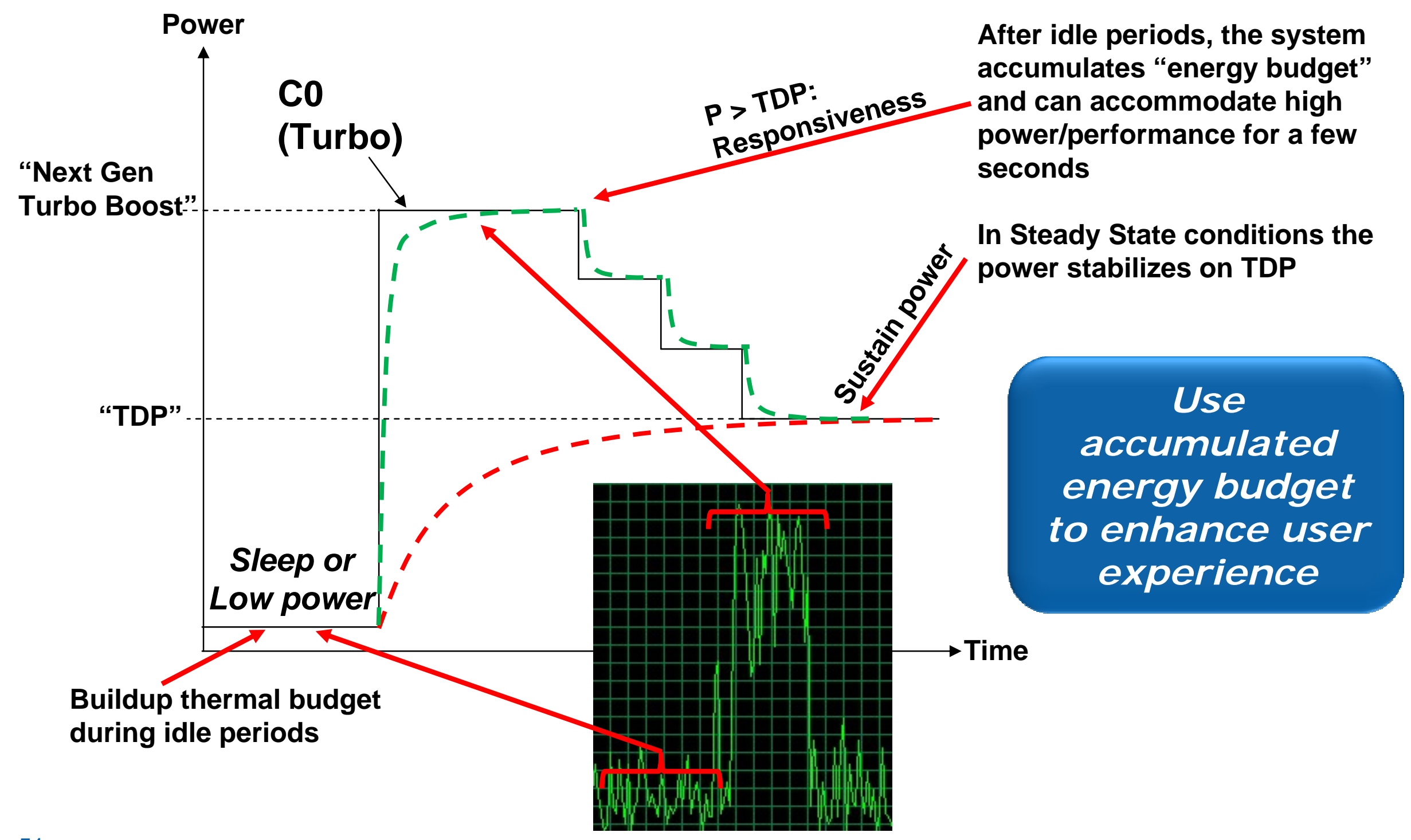




\section{Core and Graphic Power Budgeting}

- Cores and Graphics integrated on the same die with separate voltage/frequency controls; tight HW control

- Full package power specifications available for sharing

- Power budget can shift between Cores and Graphics

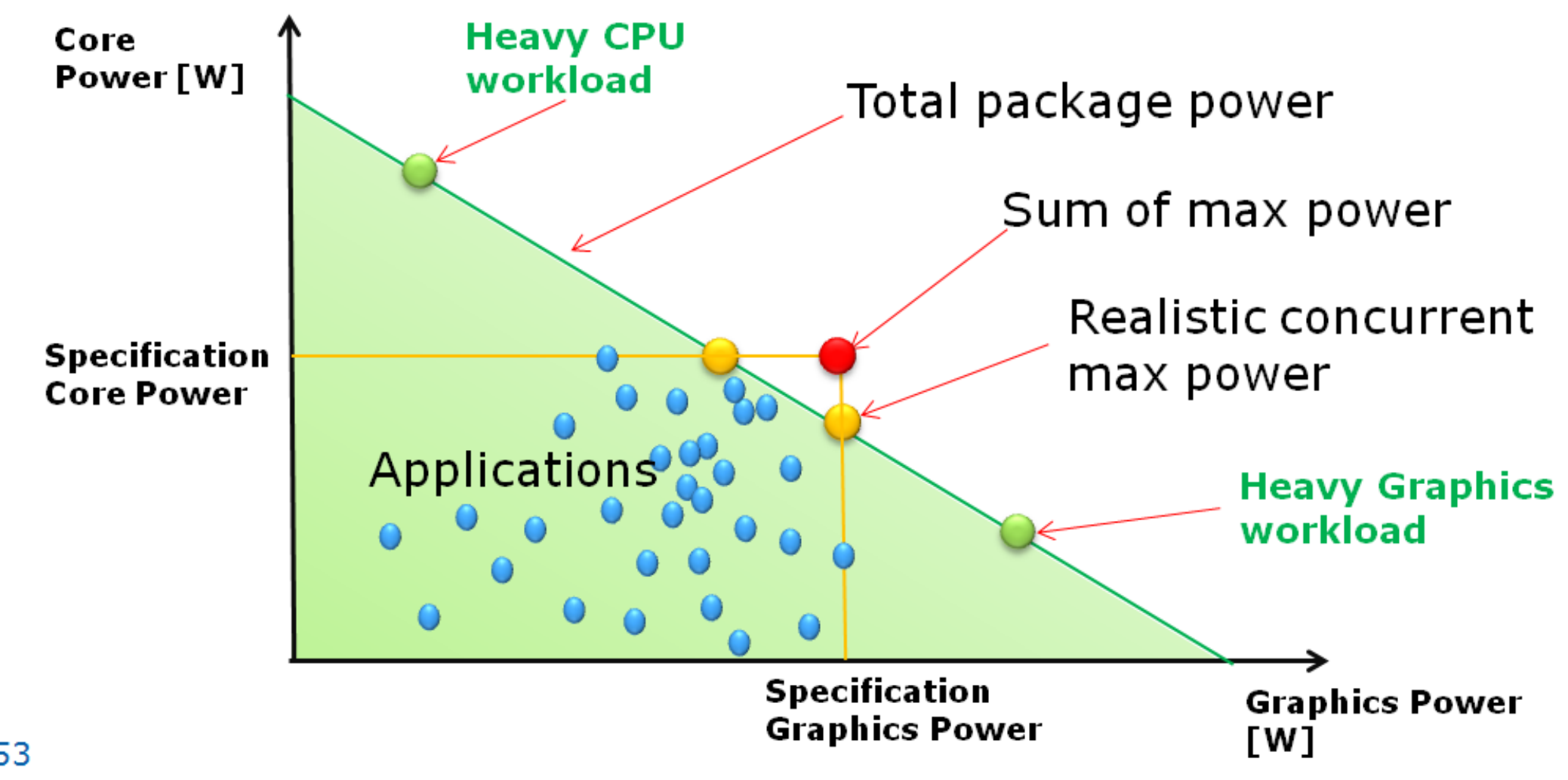




\section{Core and Graphic Power Budgeting}

- Cores and Graphics integrated on the same die with separate voltage/ frequency controls; tight HW control

- Full package power specifications available for sharing

- Power budget can shift between Cores and Graphics

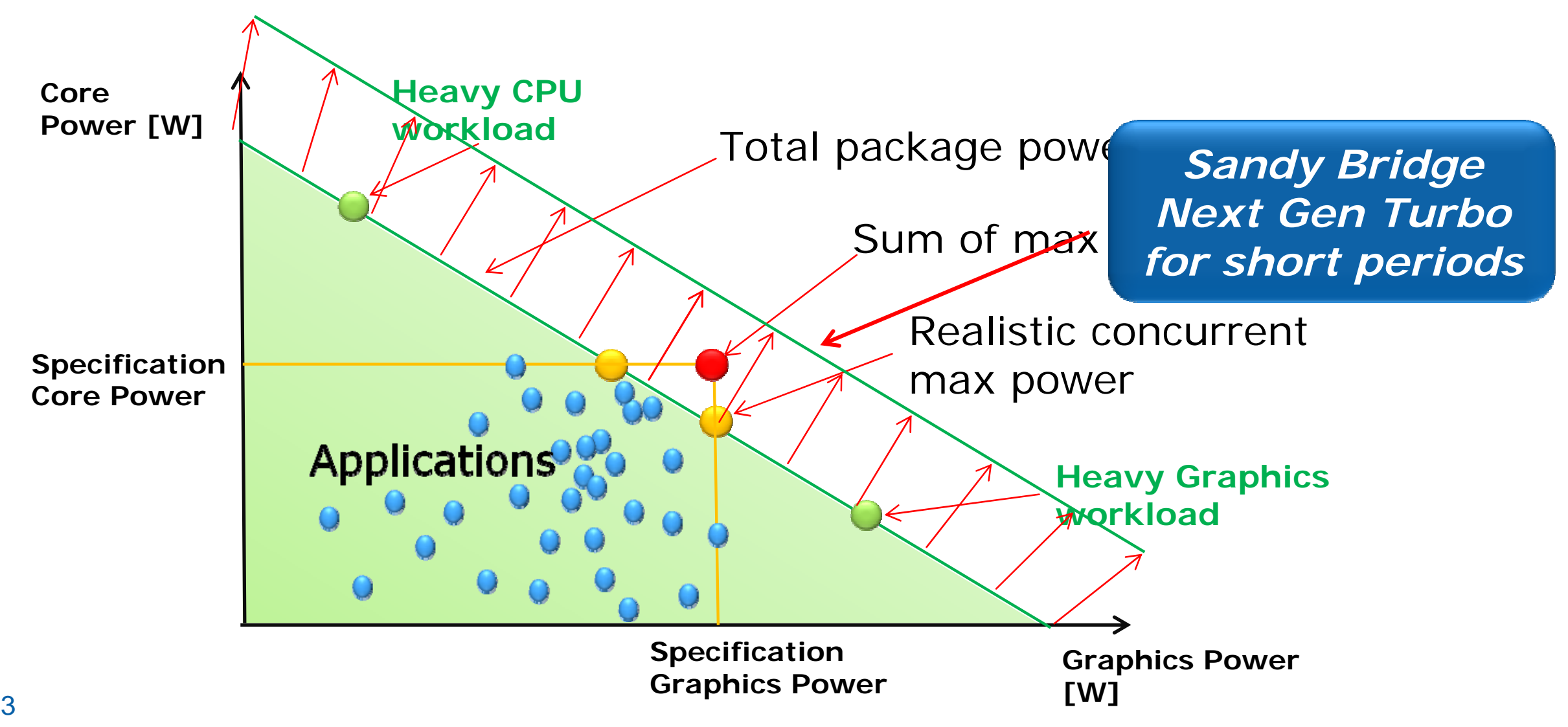




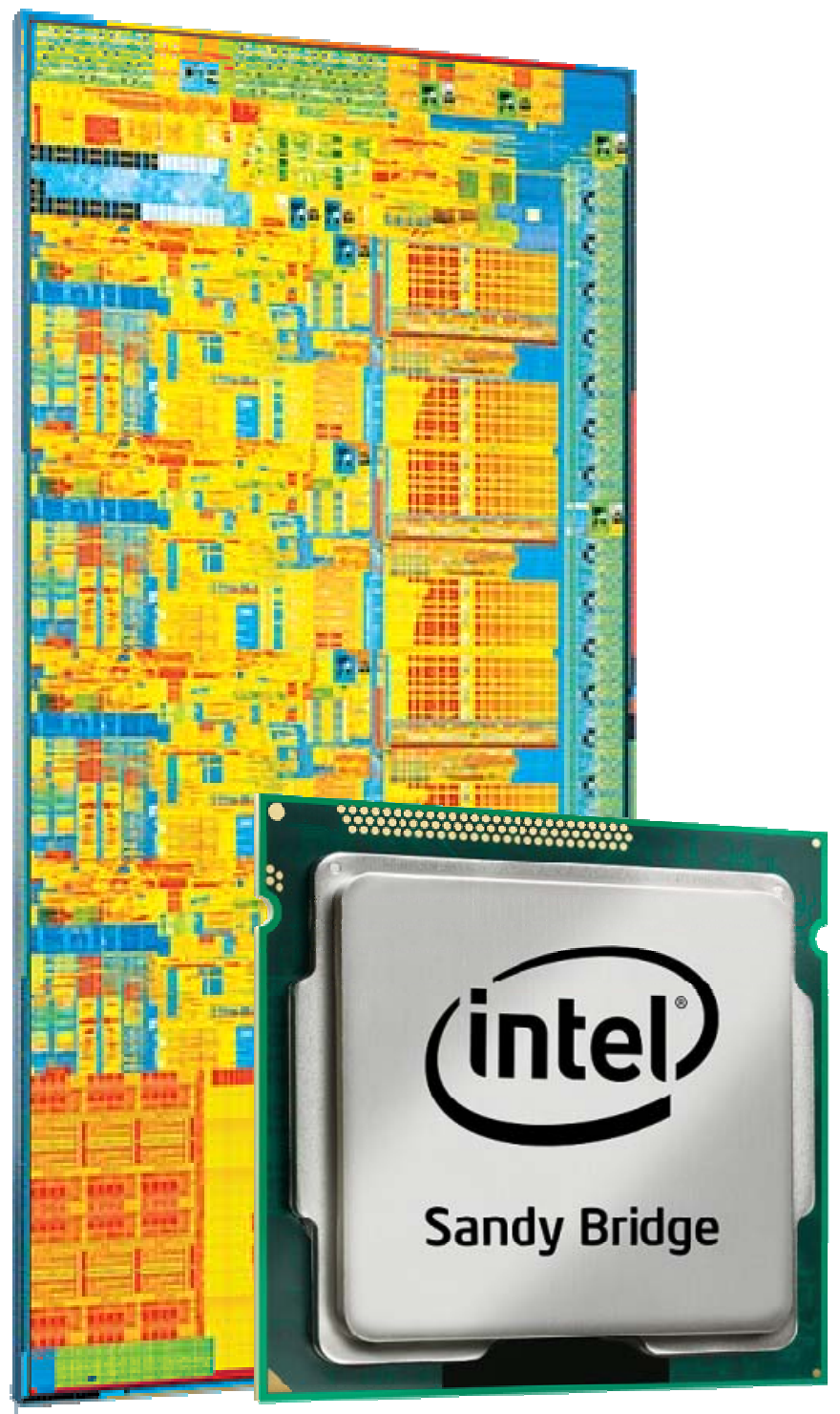

\section{Summary}

32nm Next Generation Microarchitecture Processor Graphics

System Agent, Ring Architecture and Other Innovations

Performance and Power Efficiency 